\title{
An account of the taxonomy and distribution of Syllidae (Annelida, Polychaetes) in the eastern Mediterranean, with notes on the genus Prosphaerosyllis San Martín, I 984 in the Mediterranean
}

\author{
Sarah Faulwetter ${ }^{1,4}$, Georgios Chatzigeorgiou ${ }^{2,4}$, Bella S. Galil ${ }^{3}$, \\ Christos Arvanitidis ${ }^{4}$
}

I Department of Zoology - Marine Biology, Faculty of Biology, National and Kapodestrian University of Athens, Panepistimiopolis, 15784, Athens, Greece 2 Department of Biology, University of Crete, $71409 \mathrm{He}-$ raklion, Crete, Greece 3 National Institute of Oceanography, Israel Oceanographic and Limnological Research, POB 8030, Haifa 31080, Israel 4 Institute of Marine Biology and Genetics, Hellenic Centre for Marine Research, 71003 Heraklion, Crete, Greece

Corresponding author: Sarah Faulwetter (sarifa@hcmr.gr)

Academic editor: V. Smith | Received 27 September 2011 | Accepted 23 November 2011 | Published 28 November 2011

Citation: Faulwetter S, Chatzigeorgiou G, Galil BS, Arvanitidis C (2011) An account of the taxonomy and distribution of Syllidae (Annelida: Polychaetes) in the eastern Mediterranean, with notes on the genus Prosphaerosyllis San Martín, 1984 in the Mediterranean. In: Smith V, Penev L (Eds) e-Infrastructures for data publishing in biodiversity science. ZooKeys 150: 281-326. doi: 10.3897/zookeys.150.2146

\begin{abstract}
The syllid fauna of three locations in Crete and Israel (eastern Mediterranean Sea) was studied, yielding 82 syllid species, many of which were found for the first time in the respective areas: Seventeen species were recorded for the first time on the Israeli coasts and 20 in Greek waters. Perkinsyllis augeneri (Hartmann-Schröder, 1979) and Prosphaerosyllis chauseyensis Olivier et al., 2011 are new records for the Mediterranean Sea. Detailed information is given on the morphology, ecology and distribution of the species recorded for the first time in the studied areas. In addition, an update on the distribution of the genus Prosphaerosyllis San Martín, 1984 in the Mediterranean is given and an identification key to the Mediterranean species is provided.
\end{abstract}

Copyright Sarah Faulwetter et al. This is an open access article distributed under the terms of the Creative Commons Attribution License, which permits unrestricted use, distribution, and reproduction in any medium, provided the original author and source are credited. 


\section{Keywords}

Polychaetes, Syllidae, eastern Mediterranean Sea, taxonomy, distribution, new records, alien species

\section{Introduction}

The Syllidae are a highly diverse family of polychaetes with currently around 900 valid species belonging to over 80 genera (pers. obs.) and have recently received considerable taxonomic and phylogenetic research effort, including a high number of new taxon descriptions (e.g. Aguado et al. 2007, Aguado and San Martín 2009, De Matos Nogueira et al. 2001, San Martín 2005, 2008, San Martín and Hutchings 2006, San Martín et al. 2009). Syllids are (usually) small-sized polychaetes with a high diversity of morphological and ecological features and are found globally on all types of substrates from the intertidal to the abyss (San Martín 2003).

The present study contributes to the current knowledge of the syllid fauna of three different locations in the eastern Mediterranean Sea: two in Crete, one in Israel. The material has been collected in the framework of two different research programmes and from two different habitats (Fig. 1, Table 1): a) hard-bottom samples from Crete have been obtained within the NaGISA project (Natural Geography in Shore Areas, http://www.nagisa.coml.org), a field project of the Census of Marine Life (COML, http://www.coml.org); b) soft-sediment samples from the Israeli coast have been obtained in the framework of a project focusing on the soft bottom benthos of Haifa Bay. In all samples, Syllidae were highly abundant and yielded many species recorded for the first time in the respective area, as well as a species new to science (Faulwetter et al. 2011).

In the Mediterranean Sea, syllids have been studied by numerous authors in extensive taxonomic and biogeographic works (e.g. Ben-Eliahu 1977a, 1977b, Campoy 1982, Çinar 1999, San Martín 1984b, 2003, Musco and Giangrande 2005), however, most research on the taxon is being carried out in the western Mediterranean basin, whereas the syllid fauna of the eastern Mediterranean has only recently started to be investigated more intensely (e.g. Ben-Eliahu 1977a, 1977b, Çinar 1999, Çinar and Ergen 2002, 2003, Çinar et al. 2003, Aguado and San Martín 2007, Abd-Elnaby and San Martín 2010, 2011). In Greece, polychaetes have been studied by various authors (e.g. Bellan 1964, Fassari 1982, Arvanitidis 1994, 2000, Simboura 1996, Simboura and Nicolaidou 2001, Antoniadou et al. 2004). However, the only studies in the Aegean Sea focussing specifically on Syllidae are those of Çinar (1999) and Çinar and Ergen (2002) from the Turkish Aegean coasts. Polychaetes of the Mediterranean coast of Israel have been studied by Monro (1937), Tebble (1959), Fauvel (1955, 1957), Ben-Eliahu (1976a, 1976b), Ben-Eliahu and Golani (1990) and Ben-Eliahu and Fiege (1995) and syllids in particular by Harlock and Laubier (1966) and Ben-Eliahu (1977a, 1977b). 
Table I. Sampling stations and their characteristics

\begin{tabular}{|c|c|c|c|c|}
\hline Station Code & Location & Coordinates & Depth & Habitat \\
\hline ALA-IL-1 & $\begin{array}{l}\text { Haifa Bay, } \\
\text { Israel }\end{array}$ & $\begin{array}{l}32^{\circ} 53.792^{\prime} \mathrm{N} \\
35^{\circ} 03.928^{\prime} \mathrm{E}\end{array}$ & $13.1 \mathrm{~m}$ & Fine to medium sand \\
\hline ALA-IL-2 & $\begin{array}{l}\text { Haifa Bay, } \\
\text { Israel }\end{array}$ & $\begin{array}{l}32^{\circ} 54.052^{\prime} \mathrm{N} \\
35^{\circ} 03.905^{\prime} \mathrm{E}\end{array}$ & $13.9 \mathrm{~m}$ & Sand of mixed grain sizes \\
\hline ALA-IL-5 & $\begin{array}{l}\text { Haifa Bay, } \\
\text { Israel }\end{array}$ & $\begin{array}{l}32^{\circ} 54.259^{\prime} \mathrm{N} \\
35^{\circ} 04.160^{\prime} \mathrm{E}\end{array}$ & $11.4 \mathrm{~m}$ & Silty sand \\
\hline ALA-IL-7 & $\begin{array}{l}\text { Haifa Bay, } \\
\text { Israel }\end{array}$ & $\begin{array}{l}32^{\circ} 54.544^{\prime} \mathrm{N} \\
35^{\circ} 04.093^{\prime} \mathrm{E}\end{array}$ & $10.5 \mathrm{~m}$ & Sand of mixed grain sizes with silt \\
\hline ALA-IL-8 & $\begin{array}{l}\text { Haifa Bay, } \\
\text { Israel }\end{array}$ & \begin{tabular}{|l|}
$32^{\circ} 55^{\prime} \mathrm{N}$ \\
$35^{\circ} 04.239^{\prime} \mathrm{E}$
\end{tabular} & $7.8 \mathrm{~m}$ & Coarse sand with silt \\
\hline ALA-IL-9 & $\begin{array}{l}\text { Haifa Bay, } \\
\text { Israel }\end{array}$ & $\begin{array}{l}32^{\circ} 54.518 ' \mathrm{~N} \\
35^{\circ} 03.950^{\prime} \mathrm{E}\end{array}$ & $8.7 \mathrm{~m}$ & Coarse sand \\
\hline ALA-IL-10 & $\begin{array}{l}\text { Haifa Bay, } \\
\text { Israel }\end{array}$ & $\begin{array}{l}32^{\circ} 52.509^{\prime} \mathrm{N} \\
35^{\circ} 03.520^{\prime} \mathrm{E}\end{array}$ & $12.8 \mathrm{~m}$ & Medium to coarse sand \\
\hline $\begin{array}{l}\text { CALA-1, } \\
\text { CALB-1 }\end{array}$ & $\begin{array}{l}\text { Alykes, Crete, } \\
\text { Greece }\end{array}$ & $\begin{array}{l}35^{\circ} 24.95^{\prime} \mathrm{N} \\
24^{\circ} 59.25^{\prime} \mathrm{E}\end{array}$ & $1 \mathrm{~m}$ & Cystoseira spp., Fucus virsoides \\
\hline $\begin{array}{l}\text { CALA-5, } \\
\text { CALB-5 }\end{array}$ & $\begin{array}{l}\text { Alykes, Crete, } \\
\text { Greece }\end{array}$ & $\begin{array}{l}35^{\circ} 24.95^{\prime} \mathrm{N} \\
24^{\circ} 59.25^{\prime} \mathrm{E}\end{array}$ & $5 \mathrm{~m}$ & $\begin{array}{l}\text { Filamentous Chlorophyceae, Amphiroa sp., } \\
\text { Padina pavonica }\end{array}$ \\
\hline $\begin{array}{l}\text { CALA-10, } \\
\text { CALB-10 }\end{array}$ & $\begin{array}{l}\text { Alykes, Crete, } \\
\text { Greece }\end{array}$ & $\begin{array}{l}35^{\circ} 24.95^{\prime} \mathrm{N} \\
24^{\circ} 59.25^{\prime} \mathrm{E}\end{array}$ & $10 \mathrm{~m}$ & Cystoseira spp., filamentous Chlorophyceae \\
\hline $\begin{array}{l}\text { CALA-15, } \\
\text { CALB-15 }\end{array}$ & $\begin{array}{l}\text { Alykes, Crete, } \\
\text { Greece }\end{array}$ & $\begin{array}{l}35^{\circ} 24.95^{\prime} \mathrm{N} \\
24^{\circ} 59.25^{\prime} \mathrm{E}\end{array}$ & $15 \mathrm{~m}$ & $\begin{array}{l}\text { Filamentous Chlorophyceae, filamentous } \\
\text { Phaeophyceae }\end{array}$ \\
\hline $\begin{array}{l}\text { CALA-20, } \\
\text { CALB-20 }\end{array}$ & $\begin{array}{l}\text { Alykes, Crete, } \\
\text { Greece }\end{array}$ & $\begin{array}{l}35^{\circ} 24.95^{\prime} \mathrm{N} \\
24^{\circ} 59.25^{\prime} \mathrm{E}\end{array}$ & $20 \mathrm{~m}$ & $\begin{array}{l}\text { Filamentous Phaeophyceae, Bryopsis sp., } \\
\text { Caulerpa spp. }\end{array}$ \\
\hline $\begin{array}{l}\text { CELA-1, } \\
\text { CELB-1 }\end{array}$ & \begin{tabular}{|l|} 
Elounda, \\
Crete, Greece
\end{tabular} & $\begin{array}{l}35^{\circ} 15.1^{\prime} \mathrm{N} \\
25^{\circ} 45.5^{\prime} \mathrm{E}\end{array}$ & $1 \mathrm{~m}$ & $\begin{array}{l}\text { Jania sp., Dasycladus clavaeformis, Porifera } \\
\text { spp., Litophyllum sp. }\end{array}$ \\
\hline $\begin{array}{l}\text { CELA-5, } \\
\text { CELB-5 }\end{array}$ & $\begin{array}{l}\text { Elounda, } \\
\text { Crete, Greece }\end{array}$ & $\begin{array}{l}35^{\circ} 15.1^{\prime} \mathrm{N} \\
25^{\circ} 45.5^{\prime} \mathrm{E}\end{array}$ & $5 \mathrm{~m}$ & $\begin{array}{l}\text { Jania sp., Dasycladus clavaeformis, } \\
\text { Litophyllum sp., Amphiroa sp. }\end{array}$ \\
\hline $\begin{array}{l}\text { CELA-10, } \\
\text { CELB-10 }\end{array}$ & $\begin{array}{l}\text { Elounda, } \\
\text { Crete, Greece }\end{array}$ & $\begin{array}{l}35^{\circ} 15.1^{\prime} \mathrm{N} \\
25^{\circ} 45.5^{\prime} \mathrm{E}\end{array}$ & $10 \mathrm{~m}$ & $\begin{array}{l}\text { Filamentous Phaeophyceae, Jania sp., } \\
\text { Porifera spp., Bryopsis sp. }\end{array}$ \\
\hline $\begin{array}{l}\text { CELA-15, } \\
\text { CELB-15 }\end{array}$ & $\begin{array}{l}\text { Elounda, } \\
\text { Crete, Greece }\end{array}$ & $\begin{array}{l}35^{\circ} 15.1^{\prime} \mathrm{N} \\
25^{\circ} 45.5^{\prime} \mathrm{E}\end{array}$ & $15 \mathrm{~m}$ & $\begin{array}{l}\text { Filamentous Phaeophyceae, Jania sp., } \\
\text { Peyssonellia sp., filamentous Chlorophyceae }\end{array}$ \\
\hline $\begin{array}{l}\text { CELA-20, } \\
\text { CELB-20 }\end{array}$ & \begin{tabular}{|l|} 
Elounda, \\
Crete, Greece
\end{tabular} & $\begin{array}{l}35^{\circ} 15.1^{\prime} \mathrm{N} \\
25^{\circ} 45.5^{\prime} \mathrm{E}\end{array}$ & $20 \mathrm{~m}$ & $\begin{array}{l}\text { Padina pavonica, filamentous } \\
\text { Chlorophyceae, Amphiroa sp. }\end{array}$ \\
\hline
\end{tabular}

This paper gives an account of the syllid species encountered in the three sampling locations and provides detailed information on the morphology, distribution and ecology of those species recorded for the first time in the respective area. Furthermore, during this study it became clear that the distribution range of the genus Prosphaerosyllis San Martín, 1984 in the Mediterranean is outdated or confused. In addition, since several new species have recently been described in this genus (Çinar et al. 2011, Olivier et al. 2011) and were also identified in the present material, an update on the distribution of the genus Prosphaerosyllis in the Mediterranean and an updated identification key are provided at the end of this paper. 


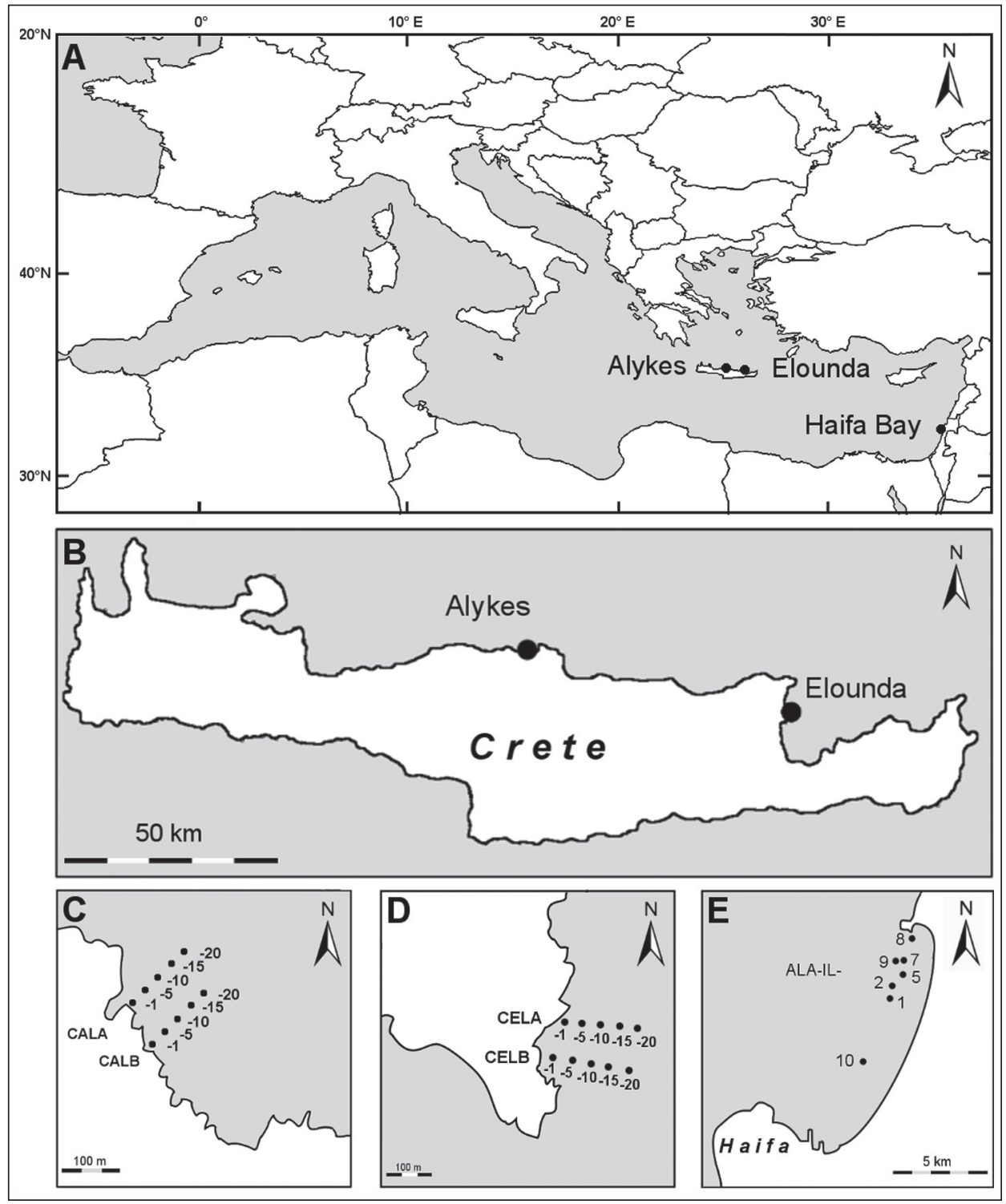

Figure I. Map of the sampling stations A Location of the stations in the Mediterranean B Locations of the two sampling stations in Crete C Alykes D Elounda E Haifa Bay.

\section{Material and methods}

\section{Specimen collection and processing}

Specimens from Israel were collected on 31 May 2009 and 11 Oct 2009 in Haifa Bay, (Israel, eastern Mediterranean Sea) from soft sediments of mixed grain sizes in shallow waters (Table 1). Sediment samples were taken with a Van-Veen grab (KAHLSICO, 
model WA265/SS214) $32 \times 35 \mathrm{~cm}$, volume $20 \mathrm{l}$, penetration $20 \mathrm{~cm}$. The sediment was preserved in buffered formalin 10\% for 3-7 days, then sieved through a $250 \mu \mathrm{m}$ mesh sieve and subsequently stored in $70 \%$ ethanol. In this study, only a subset of the collected material is presented.

Specimens from Crete were collected in September 2007 and June 2008 from two sites in northern Crete characterized by a continuous hard bottom habitat with dense algal coverage and a moderate wave exposure (Table 1). At each site, two vertical transects with sampling depths at $1 \mathrm{~m}, 5 \mathrm{~m}, 10 \mathrm{~m}, 15 \mathrm{~m}$ and $20 \mathrm{~m}$ were defined and five replicates were taken from each transect and depth. Samples were collected by means of SCUBA diving according to the NaGISA protocol (Iken and Konar 2003). A plexiglas frame $(25 \times 25 \mathrm{~cm})$ with a net of $0.5 \mathrm{~mm}$ mesh size attached to its top opening was placed onto the rock and the surface within the frame was scraped off. The sample was collected by a manually operated suction device, supplied by air from an extra scuba tank. Large particles $(>2 \mathrm{~cm})$ were collected manually after suction. The samples were subsequently washed through a $0.5 \mathrm{~mm}$ mesh sieve, fixed and preserved in $99 \%$ ethanol.

Specimens were examined under an Olympus SZx12 stereomicroscope and an Olympus BX50 microscope and identified by employing the most recent literature on Syllidae (e.g. Nygren 2004, San Martín 2003, 2005, San Martín and Hutchings 2006). Illustrations in pencil were made by means of a drawing tube, subsequently scanned, imported into a graphic program (GIMP), re-drawn and saved as a vector graphic. All specimens are deposited in the invertebrate collection of the Institute of Marine Biology and Genetics, Hellenic Centre for Marine Research. Comparative material has been loaned by the Zoologisches Museum and Institut, Universität Hamburg, Germany, Ege University, Izmir, Turkey and the Muséum National d'Histoire Naturelle, Paris, France.

Information on habitat and global distribution of species was adopted from San Martín (2003), unless indicated otherwise, and updated with findings from this study. Information on species distribution among Mediterranean regions was adopted from Musco and Giangrande (2005) and updated according to recent literature and to findings from this study. Abbreviations for biogeographic regions used in the text are: MED (Mediterranean), WB (Western Basin), EB (Eastern Basin), CB (Central Basin), AD (Adriatic Sea), AS (Aegean Sea), BS (Black Sea), LB (Levantine Basin), following Arvanitidis et al. 2002 who modified Por's (1989) system.

\section{Electronic publication}

This manuscript was prepared in a Virtual Research Environment (Scratchpads) allowing for rapid and simultaneous publication of the results in print as well as electronically in a semantically enhanced form (Blagoderov et al. 2010, Penev et al. 2010). This publication and all supplementary data (tables, figures, taxon information) are 
also available under a Creative Commons license on the Polychaete Scratchpads (http:// polychaetes.marbigen.org).

The underlying dataset of this study has been published under a Creative Commons license according to the Pensoft Data Publishing Policies and Guidelines for Biodiversity Data (Penev et al. 2011) and are available through the GBIF Integrated Publishing Toolkit hosted by Pensoft (http://ipt.pensoft.net/ipt/resource. do? $r=$ easternmedsyllids). The data are furthermore available in Darwin Core Archive format, a simple and extensible schema for sharing biodiversity data which has been developed by the Global Biodiversity Information Facility (GBIF, http:// www.gbif.org/informatics/standards-and-tools/publishing-data/data-standards/ darwin-core-archives/) to allow easy and rapid mobilisation of species occurrence data through the internet. Darwin Core Archives are essentially a set of text files stored together with an XML descriptor file which describes the structure of the data files. Data are described through the Darwin Core schema, allowing for their usage within the semantic web. This new type of data publishing allows data to be indexed and discoverable through global biodiversity infrastructures such as GBIF or other data repositories, allows data to be integrated and compared with other datasets and ensures proper accreditation of the data provider (Penev et al. 2011). Additionally, the data have been deposited in the Dryad Data Repository (http:// www.datadryad.org) and can be accessed at doi: 10.5061/dryad.4b7k408g.

\section{Results}

Examination of a total of 111 samples yielded 82 syllid species (Table 2), of which 49 were found in Alykes (Crete), 62 in Elounda (Crete) and 23 in Haifa Bay (Israel). Species of all subfamilies have been found in the stations in Crete, with the majority $(80 \%)$ of species belonging to Syllinae and Exogoninae, whereas the samples from Israel did not contain any specimens of Anoplosyllinae or Autolytinae, and $73 \%$ of the examined species belong to the small-sized Exogoninae (Fig. 2). The material yielded a number of species reported for the first time in the studied areas: Twenty species are reported for the first time in Greek waters, of these, six are new additions to the Aegean fauna. Seventeen species are newly reported for the Israeli coast, of these, 4 are also new records for the Levantine Basin. The studied material yielded also 4 species which are new additions to the eastern Mediterranean and 2 to the Mediterranean fauna (Table 2, Fig. 3). Information on morphology, distribution and ecology of the newly recorded species are given below. 


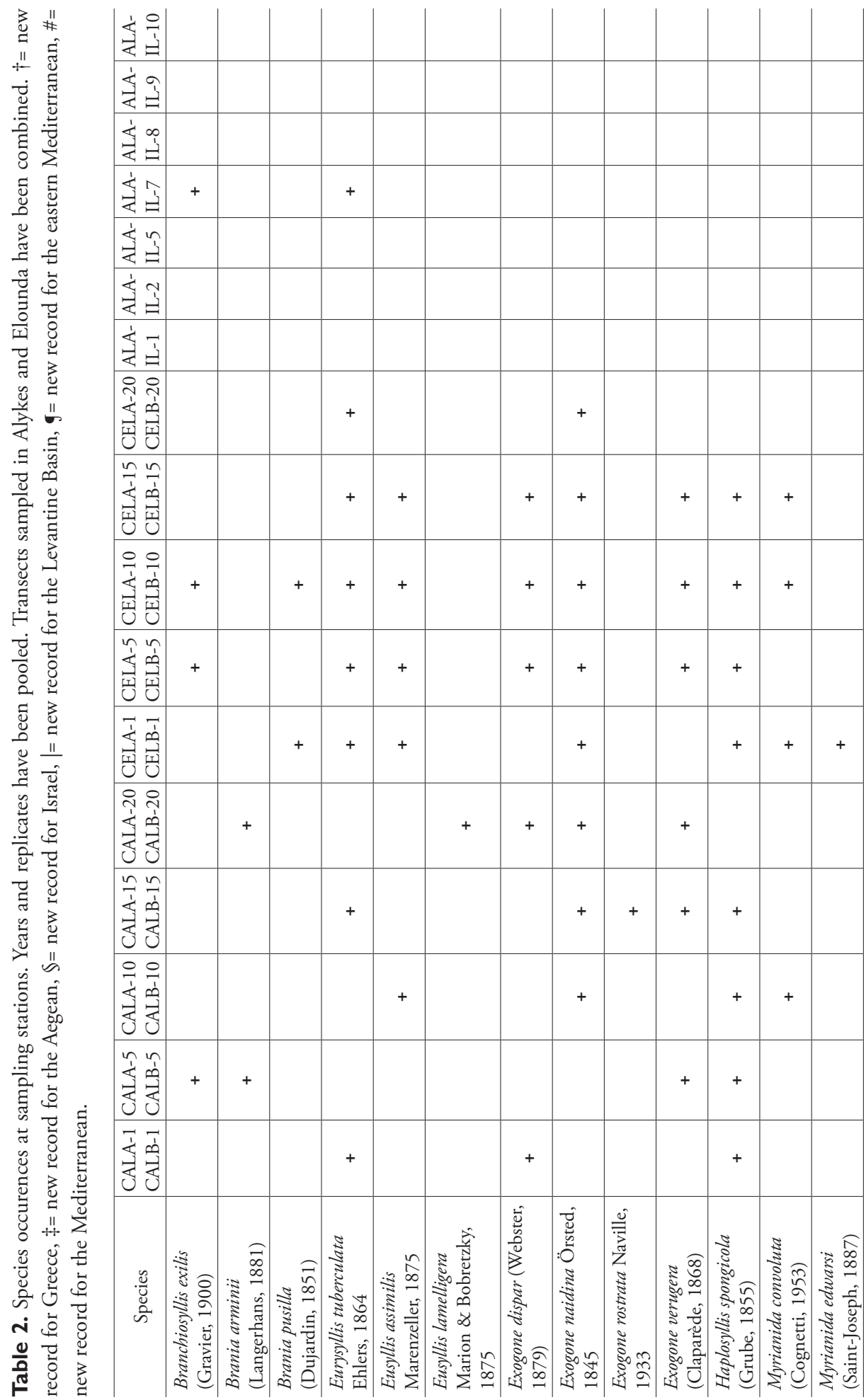




\begin{tabular}{|c|c|c|c|c|c|c|c|c|c|c|c|c|c|c|}
\hline 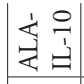 & & & & & & & & & & & + & + & & \\
\hline 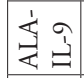 & & & & & & & & & & & & & & \\
\hline \begin{tabular}{ll}
$\dot{1}$ & $\infty$ \\
\multirow{4}{*}{} & 1 \\
\end{tabular} & & & & & & & & & & & & & & \\
\hline 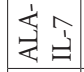 & & & & & & & & & & + & + & + & + & + \\
\hline 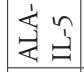 & & & & & & & & & & & & & & \\
\hline 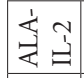 & & & & & & & & & & & & & & \\
\hline 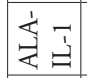 & & & & & & & & & & & & & + & \\
\hline 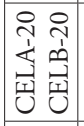 & & & & & & + & + & & + & & & & & \\
\hline 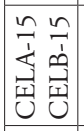 & & & & + & + & + & + & & + & & & & & \\
\hline 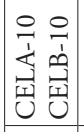 & & + & + & + & + & + & + & & + & & & & & \\
\hline 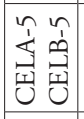 & & & + & & + & + & + & + & + & + & & & & \\
\hline 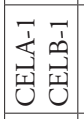 & + & + & + & + & + & & & + & & & & & & \\
\hline 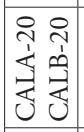 & & & & & & + & & & + & & & & & \\
\hline 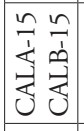 & & & & & & + & & & + & & & & & \\
\hline 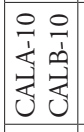 & & & + & & + & + & + & & + & & & & & \\
\hline 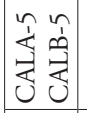 & & & & & & + & + & & + & & & & & \\
\hline 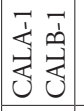 & & & + & & & & & & + & & & & & \\
\hline 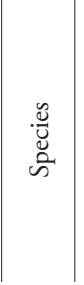 & 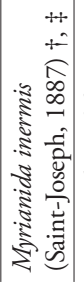 & 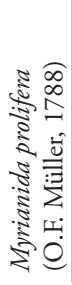 & 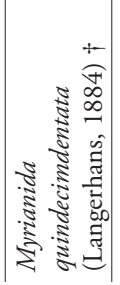 & 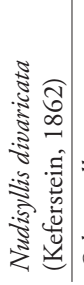 & 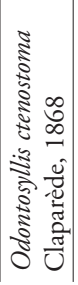 & 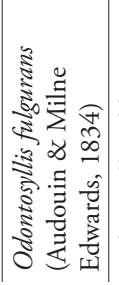 & 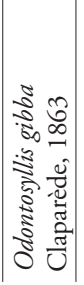 & 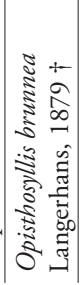 & 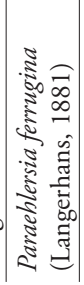 & 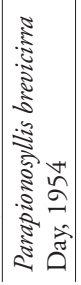 & 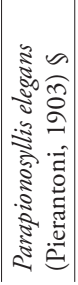 & 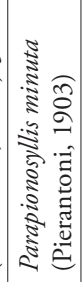 & 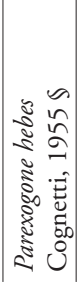 & 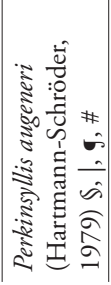 \\
\hline
\end{tabular}




\begin{tabular}{|c|c|c|c|c|c|c|c|c|c|c|c|c|}
\hline 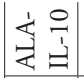 & & + & & + & & & + & & & & & \\
\hline 岁官 & & & & + & & & & & & & & \\
\hline 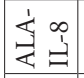 & & + & & + & & + & & & & & & \\
\hline 安念 & & + & & + & + & + & & & & & & \\
\hline 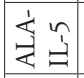 & & & & + & & & & & & & & \\
\hline 离令 & & + & & + & & + & & & & & & \\
\hline 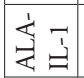 & & & & + & & & & & & & & \\
\hline 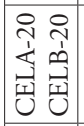 & & & & & & & & + & + & + & + & + \\
\hline 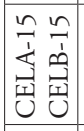 & + & & & & & & & + & + & + & + & + \\
\hline 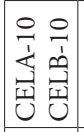 & & & + & & & & + & + & + & + & + & \\
\hline 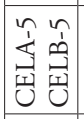 & & & & & & & + & + & + & & + & \\
\hline 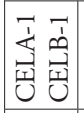 & & & & & & & & + & + & + & + & + \\
\hline 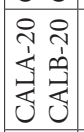 & + & & & & & & & & + & + & + & \\
\hline 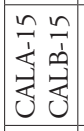 & & & & & & & & & + & & + & \\
\hline 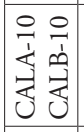 & + & & & & & & & + & + & & + & \\
\hline 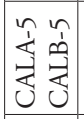 & & & & & & & & + & + & & + & \\
\hline 至预 & & & & & & & & & + & + & + & \\
\hline 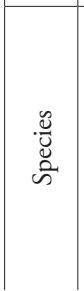 & 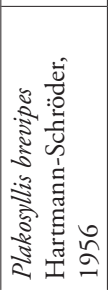 & 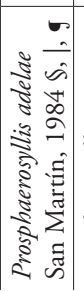 & 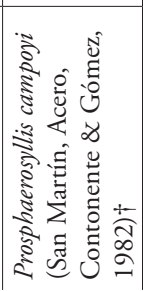 & 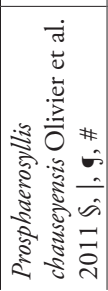 & 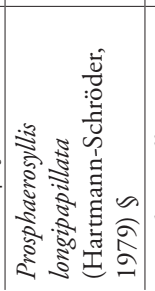 & 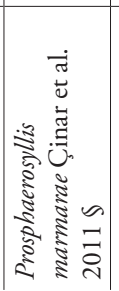 & 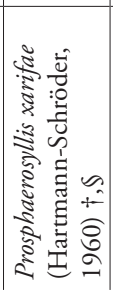 & 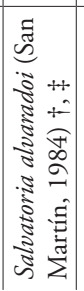 & 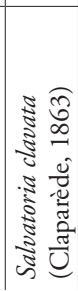 & 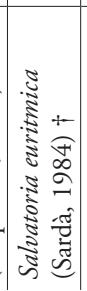 & 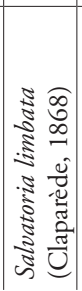 & 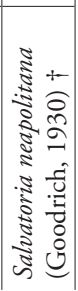 \\
\hline
\end{tabular}




\begin{tabular}{|c|c|c|c|c|c|c|c|c|c|c|c|c|c|c|c|}
\hline 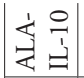 & & & & + & + & & & & & & + & & & & \\
\hline 离是 & & & & & & & & & & & & & & & \\
\hline 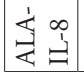 & & & & & + & + & & & & & + & & & & \\
\hline 妾令 & & & & + & + & & + & + & & + & + & + & & & \\
\hline 妾蹋 & & & & & & & & & & & & & & & \\
\hline 岁思 & & & & & & & & & & & + & & & & \\
\hline 安当 & & & & & & & & & & & + & & & & \\
\hline 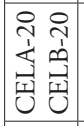 & + & + & & & & & & & + & & & & & & \\
\hline 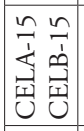 & + & + & & & + & & & & + & & & & & + & + \\
\hline 足 & + & + & + & & & & + & & + & & & & + & + & \\
\hline 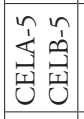 & + & + & + & & & & & & + & & & & + & + & + \\
\hline 式 & + & & & & & & + & & + & & & & & + & \\
\hline 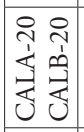 & + & + & & & & & & & + & & & & & + & \\
\hline 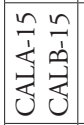 & + & + & + & & & & & & + & & & & & & \\
\hline 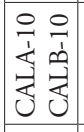 & + & + & & & & & & & + & & & & & & \\
\hline 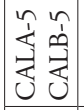 & + & & & & & & + & & + & & & & & & \\
\hline 至预 & + & & + & & & & & & + & & & & & & \\
\hline $\begin{array}{l}\mathscr{y} \\
\tilde{u} \\
\text { के }\end{array}$ & 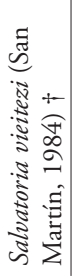 & 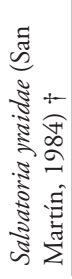 & 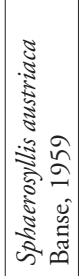 & 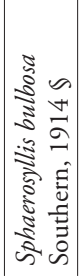 & 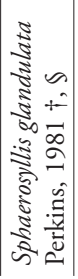 & 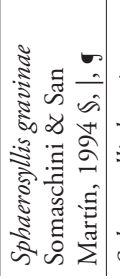 & 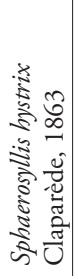 & 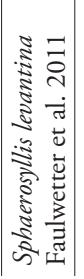 & 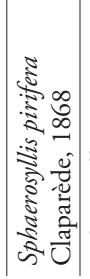 & 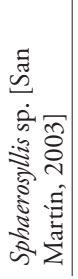 & 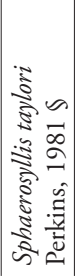 & 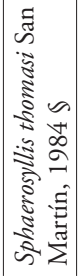 & 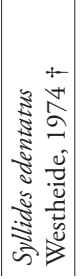 & 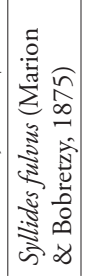 & 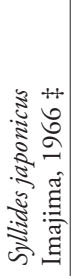 \\
\hline
\end{tabular}




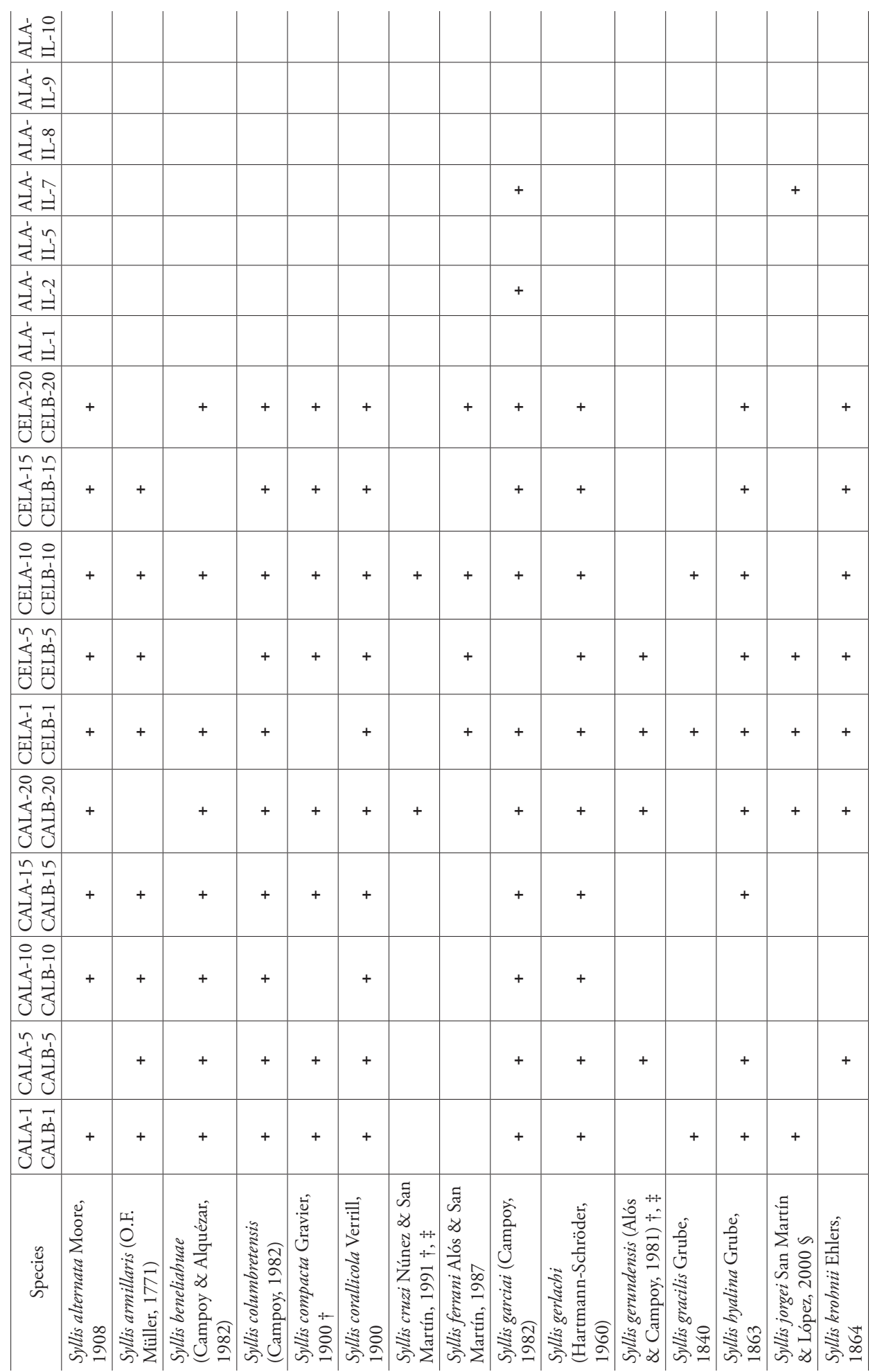




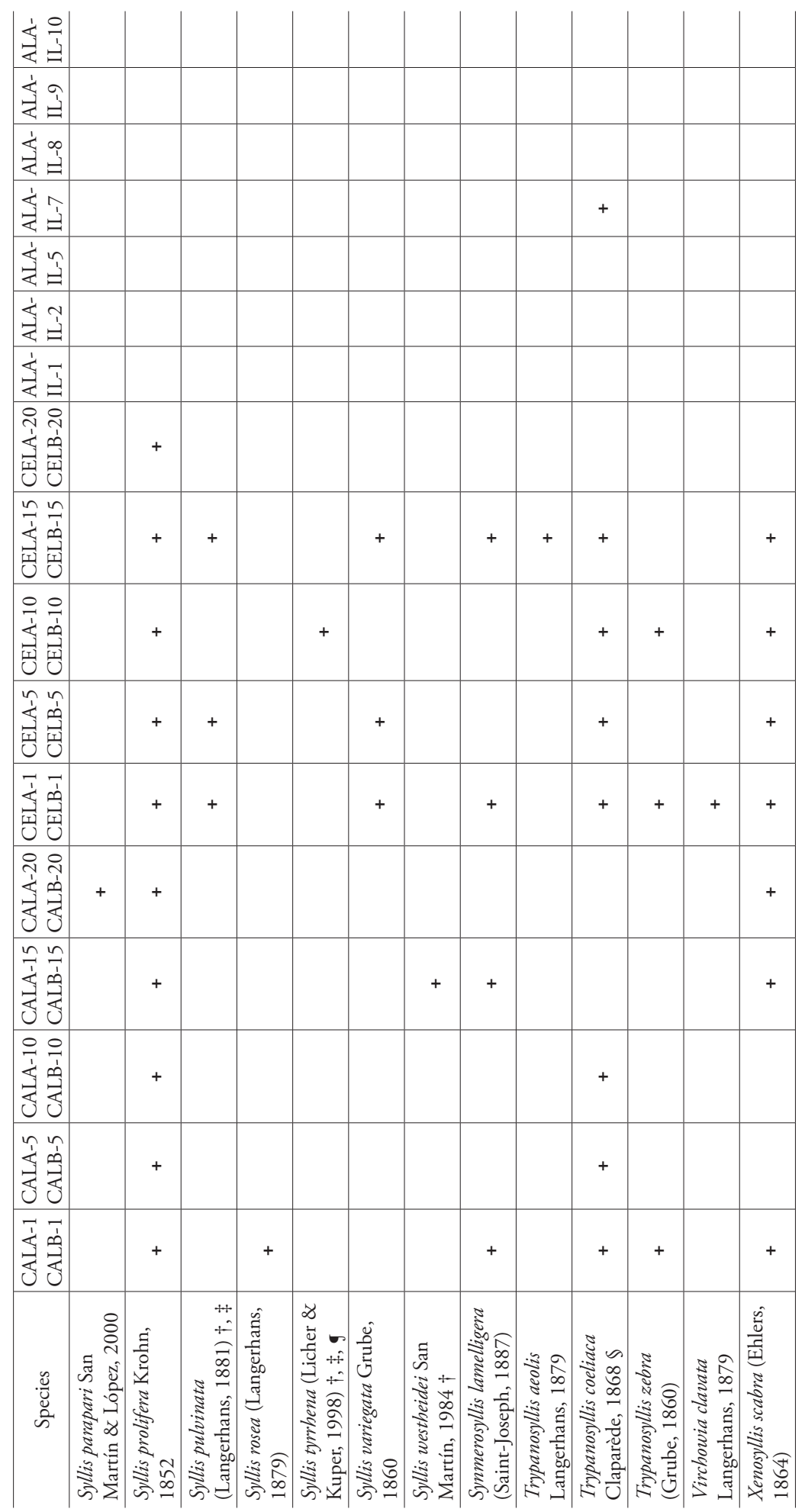




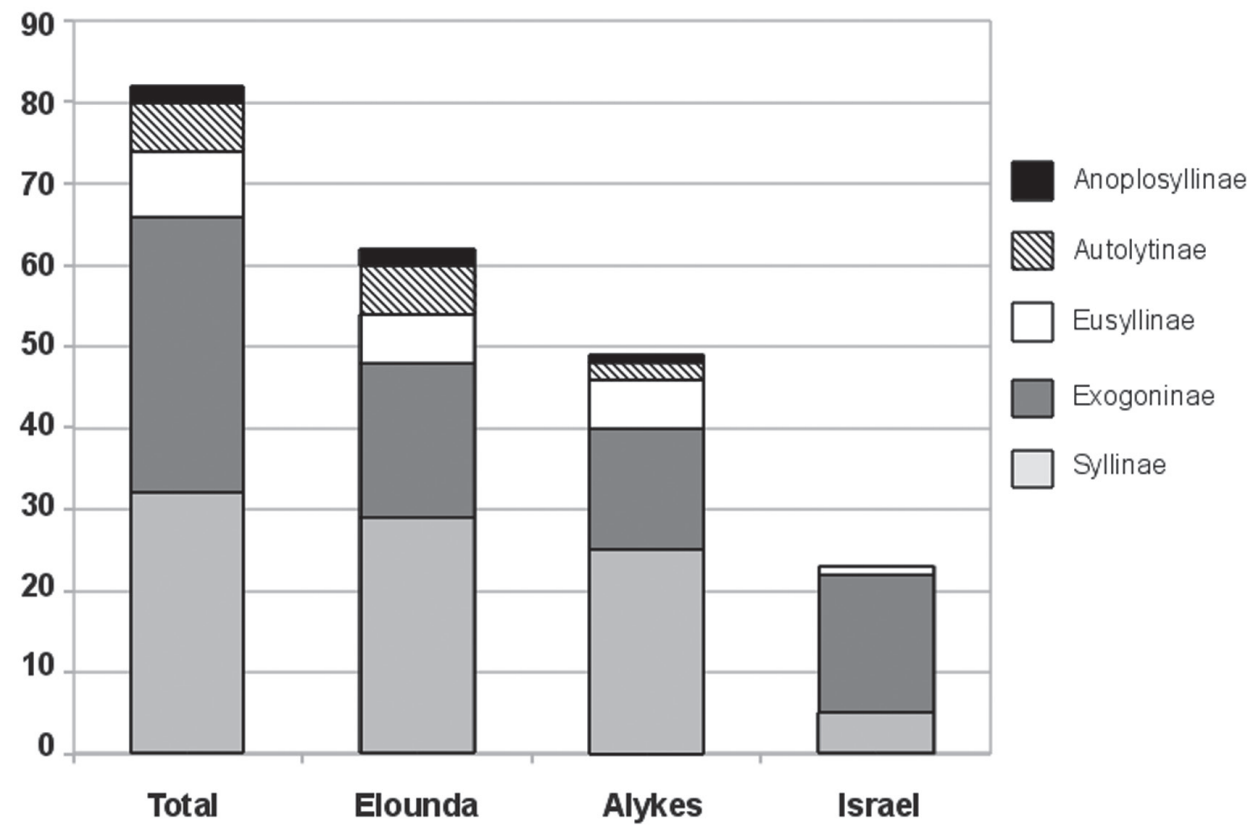

Figure 2. Numbers of species per subfamily at the three locations and in total.

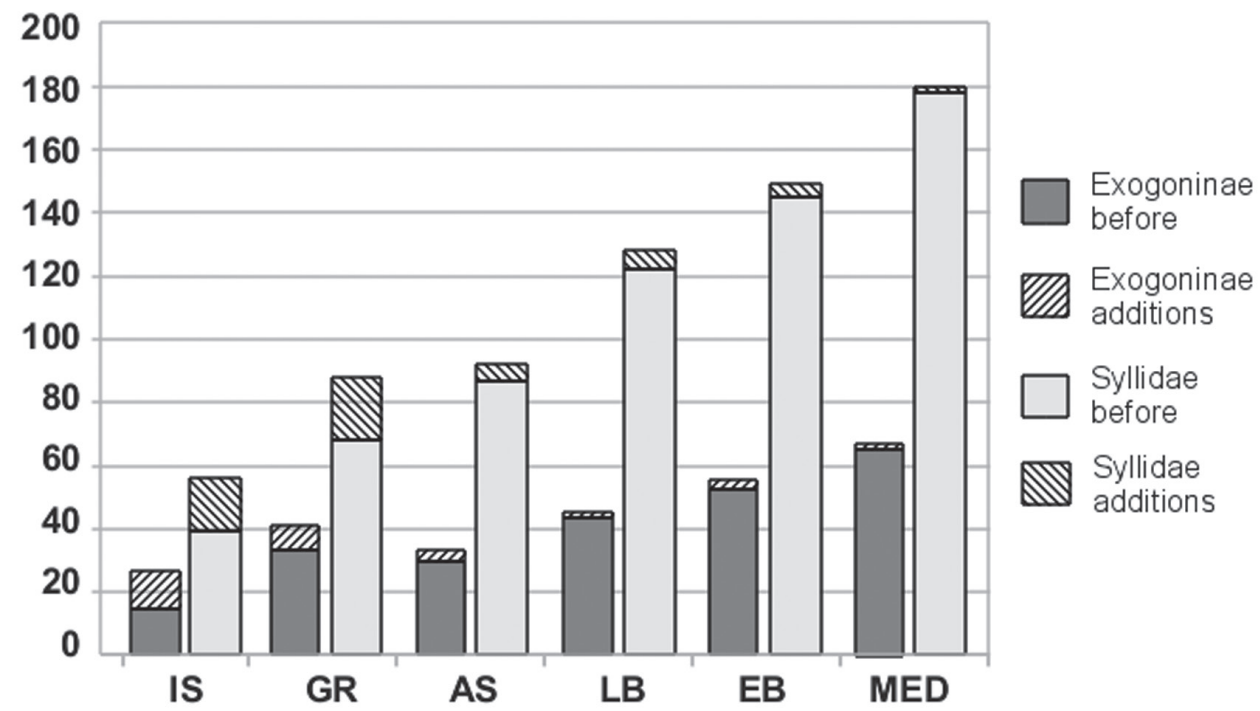

Figure 3. Numbers of additions of Syllidae and Exogoninae to various regions of the

Mediterranean. IS=Israel, GR=Greece, AS=Aegean Sea, LB=Levantine Basin, EB=Eastern Basin, MED=Mediterranean. 


\section{New records}

Subfamily Anoplosyllinae Aguado and San Martín, 2009

\section{Genus Syllides Ørsted, 1845}

Type species. Syllides longocirrata Ørsted, 1845

\section{Syllides edentatus Westheide, 1974}

http://species-id.net/wiki/Syllides_edentatus

Syllides japonica edentata Westheide, 1974a: 81, figs 36e, 37; Campoy 1982: 320; San Martín et al. 1985: 32.

Syllides edentatus: San Martín 1984b: 143, fig. 27; 2003: 143, fig. 70; Çinar 1999: 211, fig. 4.86; Çinar and Gambi 2005: 753.

Material examined. Elounda, Crete, Greece: CELA-5b-08 (2 ind.), CELA-5d-08 (2 ind.) [coll. 12.6.2008]; CELB-10c-07 (1 ind.) [coll. 27.9.2007].

Type locality. Galápagos Islands (Pacific Ocean).

Distribution. Galápagos Islands, north-east Pacific, Atlantic, Mediterranean Sea: WB, AS. New record for the Greek coast.

Habitat. Shallow subtidal depths, in sandy and muddy sediments, among photophilic algae and Zostera beds, in vermetid reefs.

\section{Syllides japonicus Imajima, 1966}

http://species-id.net/wiki/Syllides_japonicus

Syllides japonicus Imajima, 1966: 112, figs 36a-h; Banse 1971: 1477, fig. 5; San Martín 2003: 142, fig. 69; San Martín and Hutchings 2006: 360, figs 86c-f, 87a-e. Syllides cf. japonicus: San Martín 1984b: 139, fig. 26.

Material examined. Elounda, Crete, Greece: CELA-15a-07 (1 ind.) [coll. 26.9.2007]; CELA-5d-08 (1 ind.), CELB-15d-08 (1 ind.) [coll. 12.6.2008].

Type locality. Japan (Pacific Ocean).

Distribution. Japan, Australia (San Martín and Hutchings 2006), Mediterranean Sea: WB, AS, LB (Abd-Elnaby and San Martín 2010). New record for the Aegean Sea.

Habitat. Shallow subtidal depths, in sandy and muddy sediments, on rocks with algal cover, among Posidonia oceanica rhizomes. 


\section{Subfamily Autolytinae Langerhans, 1879}

\section{Genus Myrianida Milne Edwards, 1845}

Type species. Myrianida fasciata Milne Edwards, 1845

\section{Myrianida inermis (Saint-Joseph, 1887)}

http://species-id.net/wiki/Myrianida_inermis

Autolytus inermis Saint-Joseph, 1887: 237, pl. 12, fig. 117; Gidholm 1967: 193, fig.

22; Campoy 1982: 235; San Martín 1994: 274, fig. 4; 2003: 487, figs 267a, c-e;

Hartmann-Schröder 1996: 182.

Autolytus (Autolytides) inermis: Fauvel 1923: 322, figs 123h-k.

Myrianida inermis: Nygren 2004: 135, figs 65a-e.

Material examined. Elounda, Crete, Greece: CELB-1e-07 (1 ind.) [coll. 29.9.2007].

Type locality. Dinard, France (north-east Atlantic Ocean).

Distribution. North-east Atlantic, north-west Atlantic (San Martín 1994), northeast Pacific (Nygren 2004), Arctic (Ramos et al. 2010). Mediterranean Sea: WB, AS. New record for the Aegean Sea.

Habitat. Until $100 \mathrm{~m}$ depth, on rocks among algae and hydrozooans, in coralligenous substrates (Nygren 2004, San Martín 2003).

\section{Myrianida quindecimdentata (Langerhans, 1884)}

http://species-id.net/wiki/Myrianida_quindecimdentata

Autolytus quindecimdentatus Langerhans, 1884: 249, pl. 15, figs 3a-b; Gidholm 1967: 195, fig. 23; Ben-Eliahu 1977a: 86, fig. 13; Campoy 1982: 241; San Martín 1984b: 417, fig. 113; 2003: 494, figs 272a-d, 273a-b; Núnez and San Martín 1996: 213, figs 5k-m; Hartmann-Schröder 1996: 185; Çinar 1999: 63, fig. 4.8; Çinar et al. 2003: 747.

Autolytus lugens Saint-Joseph, 1887: 234, pl. 12, fig. 116; Fauvel, 1923: 318, fig. 122g; Cognetti 1961: 304.

Odontosyllis longicornis Hartmann-Schröder, 1960: 98, figs 101-104.

Myrianida quindecimdentata: Nygren 2004: 135, figs 77a-e.

Material examined. Alykes, Crete, Greece: CALA-10c-08 (4 ind.) [coll. 17.6.2008]; CALA-1b-08 (1 ind.), CALB-1c-08 (1 ind.), CALB-1d-08 (1 ind.) [coll. 18.6.2008]. Elounda, Crete, Greece: CELB-5e-07 (1 ind.) [coll. 27.9.2007]; CELB-1a-07 (2 ind.), CELA-1d-07 (2 ind.), CELB-1e-07 (5 ind.) [coll. 29.9.2007]; CELA-10b-08 (1 ind.) [coll. 11.6.2008]; CELB-1a-08 (1 ind.), CELB-1b-08 (1 ind.), CELA-5d-08 (1 ind.) [coll. 12.6.2008].

Type locality. Madeira (Atlantic Ocean). 
Distribution. East and west Atlantic (European and African coasts, Cuba), northeast Pacific, Red Sea (San Martin 1994, Nygren 2004). Mediterranean Sea: WB, CB, $\mathrm{AD}, \mathrm{AS}, \mathrm{LB}$. New record for the Greek coast.

Habitat. Subtidal depths, on biogenic calcareous substrates, among photophilic and sciaphilic algae and Posidonia oceanica rhizomes, endobiontic in sponges (Nygren 2004, San Martín 2003).

\section{Subfamily Eusyllinae Malaquin, 1893}

\section{Genus Perkinsyllis San Martín, López and Aguado, 2009}

Type species. Pionosyllis longisetosa Hartmann-Schröder, 1965

\section{Perkinsyllis augeneri (Hartmann-Schröder, 1979)}

http://species-id.net/wiki/Perkinsyllis_augeneri

Pionosyllis augeneri Hartmann-Schröder, 1979: 98, figs 119-125; 1980a: 52; 1981:

32, fig. 52 (Non Hartmann-Schröder 1991: 35); San Martín and Hutchings 2006:

326. figs $57 \mathrm{a}-\mathrm{j}, 58 \mathrm{a}-\mathrm{f}$.

Perkinsyllis augeneri: San Martín et al. 2009: 26.

Material examined. Haifa Bay, Israel: ALA-IL-7 (7 ind.) [coll. 11.10.2009].

Type locality. Boone, west Australia.

Distribution. Australia, New Zealand. Mediterranean Sea: LB. New record for the Mediterranean Sea.

Habitat. Intertidal and shallow subtidal depths, in coarse coralline sand, in muddy sand and seagrass beds (San Martín and Hutchings 2006).

Taxonomic characters. Prostomium pentagonal with 4 eyes in trapezoidal arrangement, posterior pair closer together than anterior one. Palps longer than prostomium, basally fused. Antennae cylindrical, smooth, longer than prostomium and palps. Tentacular cirri similar to antennae but slightly longer. Dorsal cirri of some anterior segments slender, longer than body width, some shorter, in midbody alternating short and long cirri, posteriorly all shorter than body width. Parapodia with 9-10 falcigers per fascicle anteriorly, 6-7 posteriorly. Shafts smooth or slightly serrated. Blades with marked dorso-ventral gradation (dorsal ones 3 times longer than ventral ones), coarsely serrated, with small subdistal tooth. After proventriculum, dorsal blades unidentate, elongated, spiniger-like, twice as long as anteriorly, ventral blades stout, with strong serration, especially basally. Dorsal simple chaeta first appearing on midbody, blunt, subdistally serrated. Ventral simple chaetae posteriorly, bidentate, equally sized teeth forming a right angle, some long spines subdistally. Paired aciculae anteriorly, single ones posteriorly, with rounded, slightly enlarged tip. Pharynx through 4 chaetigers, pharyngeal tooth located anteriorly. Proventricle through 5 chaetigers with ca. 20-22 muscle cell rows. 
Remarks. The subfamilial affiliation of Perkinsyllis augeneri has not yet been fully resolved. In recent molecular phylogenies the species groups either within Exogoninae or as a sister group, and forms a sister clade of Eusyllinae in all analyses (Aguado and Bleidorn 2010, Aguado et al. 2007).

The morphological characters of the Mediterranean individuals agree well with the description of San Martín and Hutchings (2006) from Australia. Therefore, a detailed description of the specimens is unnecessary here. The Mediterranean specimens show slight differences from the description of the Australian ones in the length of the pharynx (6-7 chaetigers in Australian specimens vs 5 in Mediterranean ones), and the number of falcigers per bundle in anterior chaetigers (ca. 15 in Australian specimens vs ca. 10 in Mediterranean ones). These differences might however be attributed to fixation and / or individual variation.

Until now, the species had been known only from north-west Australia and New Zealand, while the record from the Carribean Sea (Hartmann-Schröder 1980a) is assumed to be a different species (San Martín et al. 2009). The present findings thus extend the distribution range of the species to the eastern Mediterranean Sea. Since there are no intermediate records of the species from the Indian Ocean or Red Sea, this disjunct distribution suggests a potential human-induced introduction of the species to the Mediterranean Sea by vectors such as ballast water or fouling fauna on the hulls of ships. However, since the polychaete fauna of the Indian Ocean, Red Sea and eastern Mediterranean Sea is understudied, the species might have a truly circumtropical distribution. This is the second record of an Australian syllid species for the Mediterranean Sea (after Prosphaerosyllis longipapillata (HartmannSchröder, 1979), recorded for the first time in 2003 in Cyprus (Çinar et al. 2003)).

\section{Subfamily Exogoninae Langerhans, 1879}

\section{Genus Parapionosyllis Fauvel, 1923}

Type species. Pionosyllis gestans Pierantoni, 1903

\section{Parapionosyllis elegans (Pierantoni, 1903)}

http://species-id.net/wiki/Parapionosyllis_elegans

Pionosyllis elegans Pierantoni, 1903: 236, pl. X, fig. 2: pl. XI, fig. 27.

Parapionosyllis elegans: Fauvel 1923: 291, figs 111d-e; San Martín 1984b: 194, figs 42-43; 2003: 285, fig. 156; Çinar 1999: 127, fig. 4.40; Çinar et al. 2003: 755.

Material examined. Haifa Bay, Israel: ALA-IL-7 (11 ind.), ALA-IL-10 (45 ind.) [coll. 11.10.2009].

Type locality. Gulf of Naples (western Mediterranean Sea).

Distribution. North-east Atlantic (Iberian Peninsula). Mediterranean Sea: WB, CB, AD, AS, LB. New record for the Israeli coast.

Habitat. Until 30 m depth (San Martín 1984b), in medium to coarse sands. 


\section{Genus Prosphaerosyllis San Martín, 1984}

Type species. Sphaerosyllis xarifae Hartmann-Schröder, 1960

Prosphaerosyllis adelae San Martín, 1984

http://species-id.net/wiki/Prosphaerosyllis_adelae

Sphaerosyllis (Prosphaerosyllis) adelae San Martín, 1984a: 376, figs 1-4.

Prosphaerosyllis adelae: San Martín: 2003: 220, fig. 116.

Material examined. Haifa Bay, Israel: ALA-IL-7 (11 ind.) [coll. 31.5.2009]; ALAIL-7 (6 ind.), ALA-IL-10 (2 ind.) [coll. 11.10.2009].

Type locality. Balearic Islands (western Mediterranean Sea).

Distribution. Mediterranean Sea: WB, LB. New record for the eastern Mediterranean Sea.

Habitat. Until $13 \mathrm{~m}$ depth, in coarse sands, among Posidonia oceanica rhizomes.

Prosphaerosyllis campoyi (San Martín, Acero, Contonente and Gomez, 1982) http://species-id.net/wiki/Prosphaerosyllis_campoyi

Sphaerosyllis campoyi San Martín Acero, Contonente and Gomez, 1982: 175, fig. 2;

San Martín et al. 1985: 30, figs 3c-d; Çinar 1999: 146, fig. 4.50; Çinar et al. 2003: 756.

Sphaerosyllis (Prosphaerosyllis) campoyi: Núnez et al. 1992: 51.

Prosphaerosyllis campoyi: San Martín, 2003: 222, figs 117-118.

Material examined. Elounda, Crete, Greece: CELA-10a-07 (1 ind.) [coll. 27.9.2009]; CELA-10b-08 (1 ind.) [coll. 11.6.2008].

Type locality. Andalusia, Spain (western Mediterranean Sea).

Distribution. North-east Atlantic (Iberian Peninsula, Canary Islands), Mediterranean Sea: WB, AS, LB. New record for the Greek coast.

Habitat. Until $70 \mathrm{~m}$ depth (Çinar et al. 2003), on rocks among algae, on coralligenous substrates, in medium to coarse sands with organic material.

Remarks. The specimens agree well with the description of San Martín (2003), except for having longer dorsal papillae $(15 \mu \mathrm{m})$, especially posteriorly. 


\section{Prosphaerosyllis chauseyensis Olivier, Grant, San Martín, Archambault and McKindsey, 2011}

http://species-id.net/wiki/Prosphaerosyllis_chauseyensis

Figs $4-5$

Prosphaerosyllis chauseyensis Olivier et al., 2011, figs 1-3a, b.

Material examined. Haifa Bay, Israel: ALA-IL-8 (12 ind.) [coll. 31.5.2009]; ALA-IL-1 (23 ind.), ALA-IL-2 (4 ind.), ALA-IL-5 (1 ind.), ALA-IL-7 (73 ind.), ALA-IL-8 (24 ind.), ALA-IL-9 (68 ind.), ALA-IL-10 (99 ind.) [coll. 11.10.2009].

Comparative material examined. Sphaerosyllis brevicirra Hartmann-Schröder, 1960 (Zoological Museum Hamburg, Holotype P-17566, Ghardaqa, Red Sea: 1 individual [Label: Sphaerosyllis brevicirra n. sp., Ghardaqa (Rot. Meer) (Typ), 29.3.56, coll. Remane/Schulz]); Prosphaerosyllis chauseyensis (Muséum National d'Histoire Naturelle, Paris, Holotype MNHN POLY TYPE 1524, Chausey Islands, France: 1 individual [Label: HOLOTYPE MNHN Paris 1524, Chausey, Prosphaerosyllis sp. A, (5 ind. for SEM + Holotype), C1AM et C3AV]).

Type locality. Chausey Islands, Normandy (north-east Atlantic).

Distribution. North-east Atlantic (Normandy), Mediterranean Sea: LB. New record for the Mediterranean Sea.

Habitat. Until $13 \mathrm{~m}$ depth, in medium to very coarse sand.

Reproduction. Three specimens collected at Station ALA-IL-8 on 31 May 2009 with egg capsules attached near dorsal cirri on midbody chaetigers.

Remarks. The specimens from Israel agree well with the specimens from Normandy, however, the Mediterranean specimens differ from the Holotype in: a) Papillation pattern: each segment with one papilla between dorsal cirri and four papillae, situated dorso-laterally and ventro-laterally on each side of parapodium, most developed in posterior chaetigers, from mid-body additional papillae arranged in two very irregular lines along middle of dorsum, increasing in length towards posterior end (ca. $20 \mu \mathrm{m}$ posteriorly). Ventrally 2 smaller (about half the size of dorsal papillae) papillae in middle of ventrum at posterior end of each segment. Specimens from Normandy have an irregular papillation pattern, but papillation is more distinct laterally, as in specimens from Israel; b) Length of anal cirri: about $125 \mu \mathrm{m}$, ca. 2.5-3 times length of posterior dorsal cirri (Fig. 4) (the anal cirri are broken in the holotype and the large lateral anal papillae might have been erroneously regarded as anal cirri in the original description). Specimens from both locations have anterior dorsal cirri with two papillae (dorsal and ventral) and posterior dorsal cirri only with dorsal papilla (Fig. 5, not reported by Olivier et al. 2011).

Individuals identified by Ben-Eliahu (1977a) as Sphaerosyllis tetralix Eliason, 1920, from the Gulf of Elat and the Mediterranean Sea might in fact belong to P. chauseyensis. The description and illustrations agree with many characteristics of $P$. chauseyensis, including the characteristic papilla on the dorsal cirri. However, Ben-Eliahu reports the species to have palps widely separated anteriorly (fused in $P$. chauseyensis), dor- 


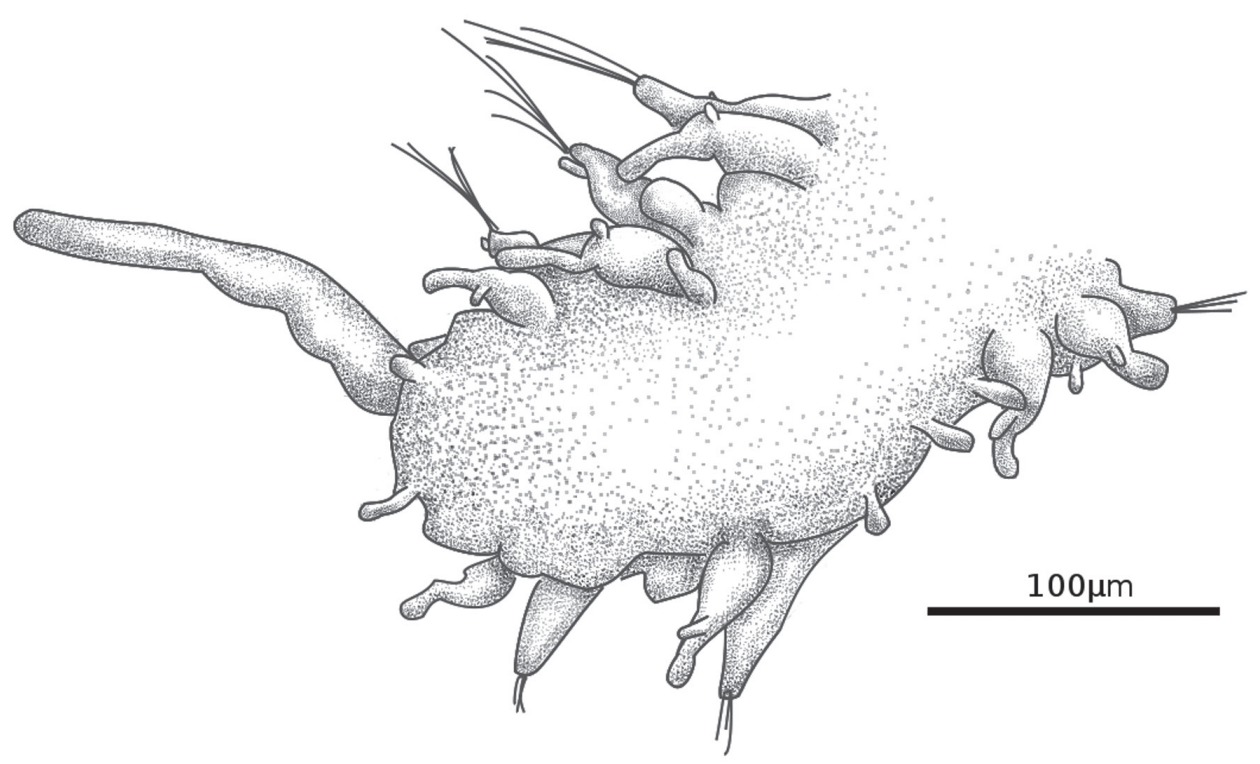

Figure 4. Prosphaerosyllis chauseyensis, pygidium (Israeli material).

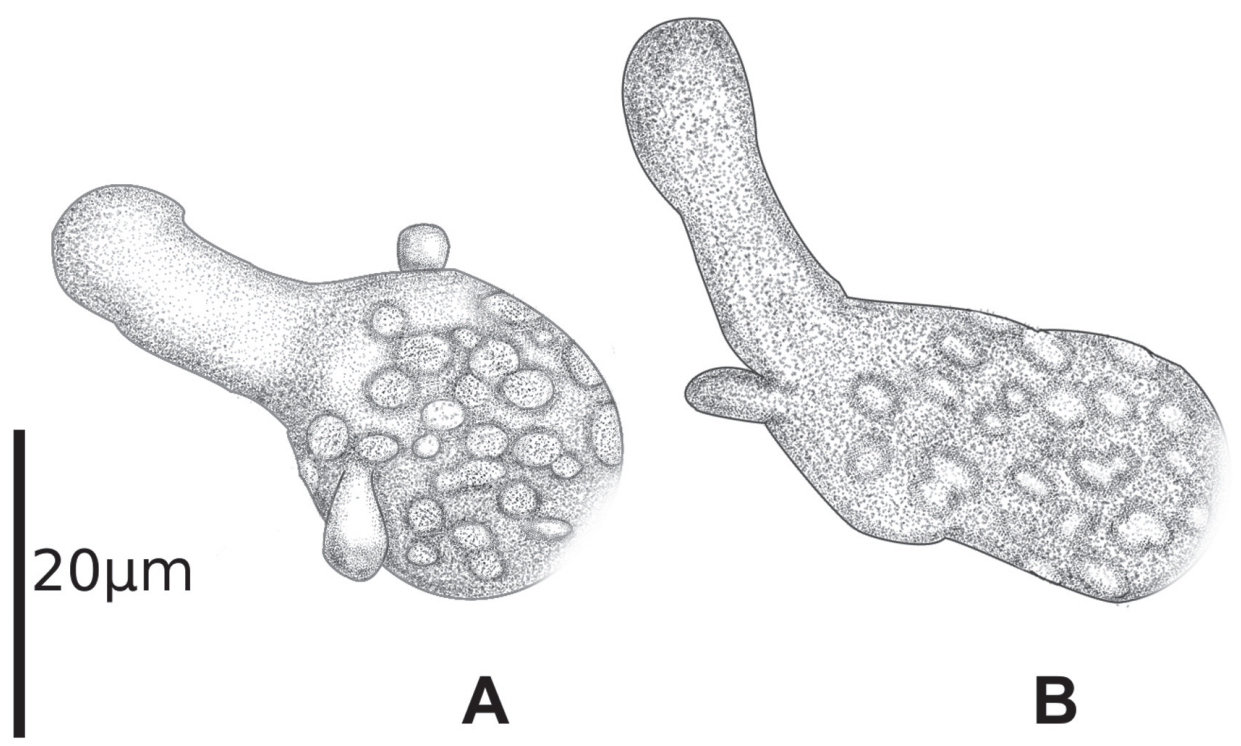

Figure 5. Prosphaerosyllis chauseyensis, anterior (A) and posterior (B) dorsal cirri (Israeli material).

sum with four longitudinal rows of papillae (irregular rows in $P$. chauseyensis) and the proventriculum stretching through 4 chaetigers ( 5 in $P$. chauseyensis). The material of the species described by Ben-Eliahu could not be examined during this study, therefore it can only tentatively be assigned to $P$. chauseyensis. 


\section{Prosphaerosyllis longipapillata (Hartmann-Schröder, 1979)}

http://species-id.net/wiki/Prosphaerosyllis_longipapillata

Sphaerosyllis longipapillata Hartmann-Schröder, 1979: 106, figs 148-150; 1982: 71;

1984: 23; 1985: 71; 1986: 43; 1991: 40; Çinar et al. 2003: 757, fig. 5.

Prosphaerosyllis longipapillata: San Martín 2005: 61, figs 17a-g, 18a-h.

Material examined. Haifa Bay, Israel: ALA-IL-7 (2 ind.) [coll. 11.10.2009].

Comparative Material examined. Prosphaerosyllis longipapillata (HartmannSchröder, 1979) (Department of Hydrobiology, Ege University, Izmir, Turkey, specimen reported in Çinar et al. 2003, Cyprus, Station D13: 1 individual [Label: P. longipapillata, Cyprus]).

Type locality. Broome, north-west Australia.

Distribution. Australia, Mediterranean Sea: LB. New record for the Israeli coast.

Habitat. Intertidal to $466 \mathrm{~m}$ depth (San Martín 2005), euryoceous, found on hard substrates with Sargassum vulgare (Çinar et al. 2003, San Martín 2005).

Remarks. The specimens from Israel agree well with the material and description of Çinar et al., 2003). However, both the material from Cyprus and Israel, as well as the description and illustrations of San Martín (2005), differ from Hartmann-Schröder's (1979) original description by the presence of dorsal papillae on the anterior chaetigers. Hartmann-Schröder (1979) reports "four long, threadlike papillae at the height of the parapodia and from chaetiger 7 onwards in pairs in a dorsal row between the parapodia". Furthermore, the Mediterranean material differs from the original description of $P$. longipapillata and from San Martín's (2005) description by having alternating rows of long and short papillae on the dorsum (Çinar et al. 2003, fig. 5). These two characteristics are reported however for Prosphaerosyllis bilineata (Kudenov and Harris, 1995) from California. To determine the identity of the Mediterranean material and whether $P$. bilineata and $P$. longipapillata are different species or not, careful examination of all type material is needed.

\section{Prosphaerosyllis marmarae Çinar, Dagli and Açik, 2011}

http://species-id.net/wiki/Prosphaerosyllis_marmarae

Prosphaerosyllis marmarae Çinar et al. 2011: 2118, figs 2-4.

Material examined. Haifa Bay, Israel: ALA-IL-2 (3 ind.), ALA-IL-8 (12 ind.) [coll. 31.5.2009]; ALA-IL-7 (4 ind.) [coll. 11.10.2009].

Comparative material examined. Prosphaerosyllis marmarae (Department of Hydrobiology, Ege University, Izmir, Turkey, Paratype: 1 individual [Label: P. marmarae, Paratype]). Prosphaerosyllis laubieri (Muséum National d'Histoire Naturelle, Paris, Holotype MNHN POLYTYPE 1525, Chausey Islands, France: 1 individual [Label: HOLOTYPE MNHN Paris 1525, Chausey B1 AM12, Prosphaerosyllis sp. B, Holotype et SEM]).

Type locality. Erdek, Marmara Sea (eastern Mediterranean). 
Distribution. Mediterranean Sea: LB, Marmara Sea. New record for the Israeli coast.

Habitat. Until $17 \mathrm{~m}$ depth, in muddy sand (Çinar et al. 2011), in coarse and mixed sand (this study).

Remarks. The specimens from Israel agree with the material of Çinar et al. (2011), except for the absence of eyespots (might be de-colourised due to fixation). The recently described $P$. laubieri Olivier et al. 2011 is very similar to $P$. marmarae. Both species have eyespots, strongly papillated palps, short, retractile antennae and dorsal cirri, pharynx and proventriculum each through 4 segments and short $(8-10 \mu \mathrm{m})$ blades of falcigers. These two species differ however in the following characteristics: a) P. laubieri has small, scattered papillae all over the dorsum, in $P$. marmarae they are restricted to the lateral margins, near the dorsal cirri; b) cirrostyles of antennae and dorsal cirri of $P$. marmarae are much shorter (1/4 of total length) than those of $P$. laubieri (1/3 of total length) and appear as small, retracted caps; c) dorsal cirri of P. laubieri possess a small papilla at distal end of cirrophore (not reported by Olivier et al. 2011); d) falcigerous blades of $P$. marmarae are stouter than those of $P$. laubieri and serrated only at their bases (serrated all along cutting edge in $P$. laubieri). P. riseri Perkins, 1981 from Florida shares with $P$. marmarae the shape of the dorsal cirri and antennae (short and strongly retracted), however, its palps are less densely papillated. Prosphaerosyllis sp. A (San Martín 1991b) from Cuba has strongly papillated palps, but no cirri on chaetiger 2 and longer dorsal cirri.

Specimens from the Red Sea described by Ben-Eliahu (1977a) as Sphaerosyllis brevicirra Hartmann-Schröder, 1960 do not belong to this species (see Discussion section), but might in fact belong to P. marmarae. The morphological characteristics of her specimens agree very well wth those of $P$. marmarae (papillated palps, presence of eyespots, minute $(19.5 \mu \mathrm{m})$, retractile cirri, falcigerous blades short $(7.8 \mu \mathrm{m})$, proventriculum longer than proboscis (through 4 segments), no discernible dorsal papillation). Differences can be found in the cutting edge of the falcigerous blades which are smooth in the Red Sea specimens, whereas those of $P$. marmarae are serrated. However, due to the size of the blades $(8 \mu \mathrm{m})$ this is a feature difficult to observe under an optical microscope and might have been overlooked. The material of the species described by Ben-Eliahu was not examined during this study, therefore it can only tentatively proposed to be assigned to $P$. marmarae.

\section{Prosphaerosyllis xarifae (Hartmann-Schröder, 1960) \\ http://species-id.net/wiki/Prosphaerosyllis_xarifae}

Sphaerosyllis xarifae Hartmann-Schröder, 1960: 103, figs 121-124; 1979: 103, figs 139-140; 1980b: 56; 1981: 37; 1984: 25; San Martín 1984b: 236, fig. 54; Çinar 1999: 166, fig. 4.62; Çinar et al. 2003: 760, fig. 6. 
Sphaerosyllis sp.: San Martín and Alvarado 1981: 224, fig. 3.

Sphaerosyllis cf. xarifae: Campoy 1982: 279.

Sphaerosyllis (Prosphaerosyllis) xarifae: Núñez et al. 1992: 51.

Prosphaerosyllis xarifae: San Martín 2003: 225, figs 119-120; 2005: 60, figs 15a-f, 16a-f; Böggemann and Westheide 2004: 435; Fukuda et al. 2009: 1448, fig. 3.

Material examined. Haifa Bay, Israel: ALA-IL-10 (5 ind.) [coll. 11.10.2009]. Elounda, Crete, Greece: CELA-10b-08 (1 ind.) [coll. 11.6.2008]; CELA-5c-08 (1 ind.) [coll. 12.6.2008].

Type locality. Sarso, Red Sea.

Distribution. Circumtropical, Mediterranean Sea: WB, CB, AS, LB. New record for both the Israeli and Greek coasts.

Habitat. Until $40 \mathrm{~m}$ depth, euryoceous, among photophilic algae, in sand, mud, seagrasses, calcareous substrates (San Martín 2005).

Remarks. Specimens from Israel agree well with the description of San Martín (2003) and Hartmann-Schröder (1960) except for having more elongated dorsal papillae, especially posteriorly $(20 \mu \mathrm{m}$, Cretan specimens: $8 \mu \mathrm{m})$.

\section{Genus Salvatoria McIntosh, 1885}

Type species. Salvatoria kerguelensis McIntosh, 1885

\section{Salvatoria alvaradoi (San Martín, 1984)}

http://species-id.net/wiki/Salvatoria_alvaradoi

Pseudobrania alvaradoi San Martín 1984b: 152, figs 28-29.

Salvatoria alvaradoi: San Martín 2003: 173, figs 87-88.

Material examined. Alykes, Crete, Greece: CALB-10b-08 (5 ind.), CALB-10d-08 (2 ind.) [coll. 17.6.2008]; CALB-5a-08 (2 ind.) [coll 18.6.2008]. Elounda, Crete, Greece: CELA-15a-07 (3 ind.), CELB-20e-07 (1 ind.) [coll. 26.9.2007], CELA10a-07 ( 1 ind.) [coll. 27.9.2007]; CELB-1a-07 (1 ind.) [coll. 29.9.2007]; CELB10a-08 (1 ind.), CELA-10b-08 (4 ind.), CELB-10b-08 (3 ind.), CELB-10c-08 (1 ind.), CELA-20a-08 (1 ind.), CELA-20d-08 (3 ind.) [coll. 11.6.2008]; CELA-5a-08 (8 ind.), CELA-5c-08 (18 ind.), CELA-5d-08 (1 ind.), CELB-15a-08 (1 ind.), CELB-15c-08 (9 ind.) [coll. 12.6.2008].

Type locality. Balearic Islands (western Mediterranean Sea).

Distribution. Mediterranean Sea: WB, CB, AS, Sea of Marmara (Karhan et al. 2008). New record for the Aegean Sea.

Habitat. Until $20 \mathrm{~m}$ depth, among algae with much sediment, among Posidonia oceanica rhizomes, in sediments with much organic material. 


\section{Salvatoria euritmica Sardá, 1984}

http://species-id.net/wiki/Salvatoria_euritmica

Pseudobrania euritmica Sardá, 1984: 10, fig. 1.

Grubeosyllis euritmica: San Martín 1991a: 718, figs 2c-d; Çinar 1999: 115, fig. 4.34;

Çinar et al. 2003: 754.

Salvatoria euritmica: San Martín 2003: 169, figs 84-86; 2005: 53, figs 8a-g.

Pionosyllis yambaensis Hartmann-Schröder, 1990: 52, figs 18-22.

Material examined. Alykes, Crete, Greece: CALB-20b-08 (1 ind.) [coll. 17.6.2008]; CALB1d-08 (4 ind.) [coll. 18.6.2008]. Elounda, Crete, Greece: CELA-15c-07 (2 ind.) [coll. 27.9.2007]; CELB-1b-07 (4 ind.), CELA-1d-07 (1 ind.) [coll. 29.9.2007]; CELA-10b-08 (1 ind.), CELA-20c-08 (1 ind.) [coll. 11.6.2008]; CELB-15d-08 (1 ind.) [coll. 12.6.2008].

Type locality. Strait of Gibraltar (western Mediterranean Sea).

Distribution. Caribbean Sea, Australia, north-east Atlantic (Iberian Peninsula, Canary Islands), Mediterranean Sea: WB, AS, LB. New record for the Greek coast.

Habitat. Until $20 \mathrm{~m}$ depth, on hard substrates between algae, in seagrass beds, on coralligenous substrates.

Remarks. Pionosyllis yambaensis was synonymized with Salvatoria euritmica by San Martín (2005) based on examination of type material.

\section{Salvatoria neapolitana (Goodrich, 1930)}

http://species-id.net/wiki/Salvatoria_neapolitana

Pionosyllis neapolitana Goodrich, 1930: 651, figs 1-12.

Pseudobrania neapolitana San Martín 1984b: 160, figs 31-32.

Grubeosyllis neapolitana: Jiménez et al. 1994: 52 figs 1-2; Böggemann and Westheide 2004: 430.

Salvatoria neapolitana: San Martín 2003: 182, fig. 94.

Pionosyllis subterranea Hartmann-Schröder, 1956: 89 figs 6-9.

Brania subterranea: Westheide 1974a: 10, fig. 6; 1974b: 87, figs 10, 42d-f.

Grubeosyllis subterranea: Núñez et al. 1992: 45.

Material examined. Elounda, Crete, Greece: CELA-15a-07 (2 ind.), CELB-20c-07 (2 ind.) [coll. 26.9.2007]; CELB-15a-08: (5 ind.), CELB-15c-08 (1 ind.) [coll. 11.6.2008]; CELB-1d-08 (1 ind.) [coll. 12.6.2008].

Type locality. Bay of Naples, Italy (western Mediterranean Sea).

Distribution. Circumtropical, Mediterranean Sea: WB, AS (Çinar et al. 2008). New record for the Greek coast.

Habitat. Until $20 \mathrm{~m}$ depth, in coarse sand, among photophilic algae. 
Remarks. Pionosyllis subterranea was synonymized with $P$. neapolitana and transferred to Grubeosyllis by Jiménez et al. (1994). San Martín (2003) subsequently replaced the name Grubeosyllis with Salvatoria, which has priority over the former.

\section{Salvatoria vieitezi (San Martín, 1984)}

http://species-id.net/wiki/Salvatoria_vieitezi

Pseudobrania vieitezi San Martín, 1984b: 160, figs 31-32.

Grubeosyllis vieitezi: San Martin 1991a: 718, fig. 2e-f; Çinar 1999: 117, fig. 4.35; Çinar et al. 2003: 754; López and San Martín 1997: 105, fig 3.

Salvatoria vieitezi: San Martín 2003: 184, figs 95-96.

Material examined. Alykes, Crete, Greece: CALA-10d-08 (1 ind.), CALA-15c-08 (1 ind.), CALA-20c-08 (3 ind.), CALB-20c-08 (1 ind.), CALB-20b-08 (1 ind.) [coll. 17.6.2008]; CALA-1b-08 ( 2 ind.), CALB-1b-08 ( 1 ind.), CALB-5a-08 (1 ind.) [coll. 18.6.2008]; CALB-20e-07 (1 ind.) [coll. 18.9.2007]; CALA-5c-07 (1 ind.) [coll. 19.9.2007]. Elounda, Crete, Greece: CELA-20d-07 (3 ind.) [coll. 26.9.2007]; CELA10b-07 (1 ind.) [coll. 27.9.2007]; CELA-20c-08 (1 ind.), CELA-20d-08 (7 ind.) [coll. 11.6.2008]; CELA-5d-08 (1 ind.), CELB-15a-08 (1 ind.), CELB-1b-08 (5 ind.) [coll. 12.6.2008].

Type locality. Balearic Islands (western Mediterranean Sea).

Distribution. North-east Atlantic (Iberian Peninsula, Canary Islands), Caribbean, Mediterranean Sea: WB, CB, AS. New record for the Greek coast.

Habitat. Until $30 \mathrm{~m}$ depth, on rocky substrates among photophilic algae, as endobiont of sponges, among Posidonia oceanica rhizomes.

\section{Salvatoria yraidae (San Martín, 1984)}

http://species-id.net/wiki/Salvatoria_yraidae

Pseudobrania yraidae San Martín, 1984b: 156, fig. 30.

Grubeosyllis yraidae: Çinar 1999: 121, fig. 4.37.

Salvatoria yraidae: San Martín 2003: 163, figs 80-81.

Material examined. Alykes, Crete, Greece: CALB-10b-08 (1 ind.), CALB-15a-08 ( 1 ind.), CALB-20b-08 (3 ind.), CALB-20d-08 (1 ind.) [coll. 17.6.2008]. Elounda, Crete, Greece: CELA-15b-07 (1 ind.), CELA-15e-07 (2 ind.) [coll. 26.9.2007]; CELA-5c-07 (4 ind.) [coll. 27.9.2007]; CELA-10b-08 (3 ind.), CELB-10b-08 (8 ind.), CELB-10c-08 (1 ind.), CELA-20a-08 (1 ind.), CELA-20b-08 (1 ind.) [coll. 11.6.2008]; CELB-15a-08 (6 ind.), CELB-15c-08 (4 ind.), CELA-15d-08 (5 ind.), CELB-15d-08 (5 ind.) [coll. 12.6.2008].

Type locality. Balearic Islands (western Mediterranean Sea). 
Distribution. Mediterranean Sea: WB, CB, AD, AS. New record for the Greek coast.

Habitat. Until $20 \mathrm{~m}$ depth, in sandy substrates, on rocks among algae.

\section{Genus Sphaerosyllis Claparède, 1863}

Type species. Sphaerosyllis hystrix Claparède, 1863

\section{Sphaerosyllis bulbosa Southern, 1914}

http://species-id.net/wiki/Sphaerosyllis_bulbosa

Sphaerosyllis bulbosa Southern, 1914: 20, plates I-II, figs 2a-g; Fauvel, 1923: 304, figs.

116h-r; Cognetti 1961: 30; Rullier 1972: 69; Campoy 1982: 276; Parapar et al.

1994: 98, fig. 4; Çinar et al. 2003: 756; San Martin 2003: 191, figs 98-99.

Sphaerosyllis (Sphaerosyllis) bulbosa: Hartmann-Schröder 1996: 175.

Material examined. Haifa Bay, Israel: ALA-IL-7 (4 ind.), ALA-IL-10 (51 ind.) [coll. 11.10.2009].

Type locality. Ireland (Atlantic Ocean).

Distribution. North-east Atlantic, Arctic Sea (Ramos et al. 2010), New Caledonia (Rullier 1972). Mediterranean Sea: WB, CB, AD, AS, LB, BS (Surugiu 2005). New record for the Israeli coast.

Habitat. Until $70 \mathrm{~m}$ depth, in sandy or muddy sediments, on calcareous substrates.

Remarks. The examined material differs from the description of San Martín (2003) in having papillated palps.

\section{Sphaerosyllis glandulata Perkins, 1981}

http://species-id.net/wiki/Sphaerosyllis_glandulata

Sphaerosyllis glandulata Perkins, 1981: 1123, figs 18-19; Uebelacker 1984: 33, figs 25-26; San Martín 1991a: 232; 2003: 193, fig. 100; Men et al. 1993: 31, fig. 8;

Somaschini and San Martín 1994: 361, fig. 3; Çinar 1999: 152, fig. 4.53; San

Martín and Bone 2001: 613.

Sphaerosyllis cf. glandulata: Ding and Westheide 2008: 131, figs. 5a-h.

Material examined. Haifa Bay, Israel: ALA-IL-7 (1 ind.) [coll. 31.5.2009]; ALA-IL-7 (47 ind.), ALA-IL-10 (19 ind.) [coll. 11.10.2009]. Elounda, Crete, Greece: CELA15d-08 (1 ind.) [coll. 12.6.2008].

Type locality. Florida, Hutchinson Island. 
Distribution. West Atlantic (Florida, Caribbean Sea), China (Ding and Westheide 2008) Mediterranean Sea: WB, AD, AS, LB (Abd-Elnaby and San Martín 2010). New record for both the Israeli and Greek coasts.

Habitat. Until $120 \mathrm{~m}$ depth, in calcareous habitats and fine to coarse sands, among photophilic algae.

Remarks. The specimens from Israel differ from San Martín's (2003) description in having papillated palps and a longer proventriculum (3-4 chaetigers vs 2 chaetigers in the Iberian material). Other characteristics, especially chaetal ones, agree well with former descriptions of $S$. glandulata.

\section{Sphaerosyllis gravinae Somaschini and San Martín, 1994}

http://species-id.net/wiki/Sphaerosyllis_gravinae

Sphaerosyllis gravinae Somaschini and San Martín, 1994: 358, figs 1-2; San Martín 2003: 188, fig. 97.

Material examined. Haifa Bay, Israel: ALA-IL-8 (4 ind.) [coll. 31.5.2009].

Type locality. Zannone Island, Italy (western Mediterranean Sea).

Distribution. Mediterranean Sea: WB, AD, LB. New record for the eastern Mediterranean Sea.

Habitat. Shallow subtidal depths, in medium to coarse sands, among algae.

\section{Sphaerosyllis taylori Perkins, 1981}

http://species-id.net/wiki/Sphaerosyllis_taylori

Sphaerosyllis taylori Perkins, 1981: 1140, fig. 26; Uebelacker 1984: 29, figs 21-22; San Martín 1984b: 247, fig. 58; 2003: 206, fig. 108; Russell 1991: 71; Núnez et al. 1992: 49; Parapar et al. 1994: 99; Simboura 1996: 53, fig. 6; San Martín and Bone 2001: 614; Çinar 1999: 161, fig. 4.58; Ruíz-Ramírez and Salazar-Vallejo 2001: 131, fig. 6 (115-122); Çinar et al. 2003: 759; Liñero-Arana and Díaz-Díaz 2011 : 9 , figs $2.1-2.5$ in online material.

Material examined. Haifa Bay, Israel: ALA-IL-1 (1 ind.); ALA-IL-2 (33 ind.) [coll. 31.5.2009]; ALA-IL-7 (103 ind.), ALA-IL-10 (14 ind.) [coll. 11.10.2009].

Type locality. Florida, Hutchinson Island.

Distribution. North-east and north-west Atlantic (North Sea to Canary Islands, east coast of the U.S. to Venezuela), Pacific Ocean (Galápagos Islands) (Liñero-Arana and Díaz-Díaz 2011), Arctic Sea (Ramos et al. 2010), Mediterranean Sea: WB, CB, AD, AS, BS, LB (Abd-Elnaby and San Martín 2010). New record for the Israeli coast.

Habitat. Shallow subtidal depths, in muddy to coarse sands with organic material, on rocks among photophilic or calcareous algae, among Posidonia oceanica rhizomes. 


\section{Sphaerosyllis thomasi San Martín 1984}

http://species-id.net/wiki/Sphaerosyllis_thomasi

Sphaerosyllis thomasi San Martín, 1984b: 250, fig. 59; 2003: 199, figs 103-104; Arvanitidis 1994: 80; Çinar 1999: 163, fig. 4.60.

Material examined. Haifa Bay, Israel: ALA-IL-7 (2 ind.) [coll. 11.10.2009].

Type locality. Balearic Islands (western Mediterranean Sea).

Distribution. Mediterranean Sea: WB, CB, AD, AS, LB. New record for the Israeli coast.

Habitat. Shallow subtidal depths, in muddy to coarse sands, among Posidonia oceanica rhizomes.

Remarks. The examined specimens agree well with the description of San Martín (2003), especially in the chaeteal structures, but in the Israeli specimens the dorsal cirri are as long as parapodial lobes in posterior and midbody chaetigers and only slightly shorter than parapodial lobe in anterior chaetigers (dorsal cirri shorter than parapodial lobe in San Martín's (2003) description).

\section{Subfamily Syllinae Grube, 1850}

Genus Opisthosyllis Langerhans, 1879

Type species. Opisthosyllis brunnea Langerhans, 1879

\section{Opisthosyllis brunnea Langerhans, 1879}

http://species-id.net/wiki/Opisthosyllis_brunnea

Opisthosyllis brunnea Langerhans, 1879: 541, fig. 7; Augener 1918: 274, text-fig. 25;

Tebble 1956: 90, figs 5d-e; Day 1967: 253, figs 12.5 c-e. Hartmann-Schröder 1979: 86; 1980b: 48; 1981: 24; 1982: 58; 1991: 25, fig. 19; Fauchald 1977:20, fig. 5; San Martín 1984b: 311, figs 75-76; 2003: 330, fig. 183; Çinar 1999: 237, fig. 4.99; Amaral et al. 2005: 164, figs a-e on same page; Abd-Elnaby 2009: 15, plate $3-16$, figs $3 \mathrm{~g}-\mathrm{h}$.

Material examined. Elounda, Crete, Greece: CELA-1d-07 (1 ind.) [coll. 29.9.2007], CELA-5d-08 (1 ind.) [coll. 12.6.2008].

Type locality. Madeira (Atlantic Ocean).

Distribution. Circumtropical. Mediterranean Sea: WB, CB, AS, LB. New record for the Greek coast.

Habitat. Intertidal to shallow subtidal, on hard substrates (vermetid reefs, among photophilic algae), endobiont of sponges. 


\section{Genus Syllis Lamarck, 1818}

Type species. Syllis monilaris Lamarck, 1818

\section{Syllis alternata Moore, 1908}

http://species-id.net/wiki/Syllis_alternata

Syllis alternata Moore, 1908: 323; 1909: 321; Çinar 1999: 246, fig. 4.102; Çinar and

Gambi 2005: 754; Çinar and Ergen 2003: 777.

Typosyllis alternata: Kudenov and Harris 1995: 83, fig. 1.32; Licher 2000: 253, figs 17p, 106; Imajima 2003: 163.

Material examined. Alykes, Crete, Greece: CALB-15c-07 (1 ind.) [coll. 18.9.2007]; CALB-1a-07 (1 ind.) [coll. 19.9.2007]; CALA-10d-08 (2 ind.), CALA-15d-08 (1 ind.), CALB-20b-08 (1 ind.), CALA-20b-08 (2 ind.), CALA-20c-08 (5 ind.), CALB20d-08 (6 ind.) [coll. 17.6.2008]. Elounda, Crete, Greece: CELB-20c-07 (1 ind.) [coll. 26.9.2007]; CELB-1a-07 (4 ind.) [coll. 29.9.2007]; CELA-10b-08 (1 ind.), CELB-10b-08 (1 ind.), CELA-10c-08 (1 ind.), CELB-10c-08 (1 ind.), CELA-10d-08 ( 2 ind.), [coll. 11.6.2008]; CELB-1a-08 (1 ind.), CELA-5b-08 (1 ind.), CELA-5d-08 ( 2 ind.), CELB-15c-08 (5 ind.) [coll. 12.6.2008].

Type locality. Alaska (Pacific Ocean).

Distribution. East Pacific (Alaska to Panama), west Atlantic (North Carolina to Cuba) (Capa et al. 2001), Japan (Imajima 2003), Indonesia (Aguado et al. 2008), Mediterranean: WB, CB, AS, LB. New record for the Greek coast.

Habitat. Until $2500 \mathrm{~m}$ depth (Moore 1909), among Posidonia oceanica rhizomes, calcareous algae, corals and photophilic algae (San Martín 2003), in sandy and muddy sediments (Moore 1909).

\section{Syllis compacta Gravier, 1900}

http://species-id.net/wiki/Syllis_compacta

Syllis (Typosyllis) compacta Gravier, 1900: 165, pl. 9, fig. 11, text-figs 35-38.

Syllis compacta: López et al. 1996: 110, fig 3; Çinar 1999: 263, fig. 4.113; San Martín 2003: 433, figs 238-239.

Syllis golfonovensis: San Martín 1984b: 395, fig. 104 (Non Syllis golfonovensis Hartmann-Schröder, 1962.

Material examined. Alykes, Crete, Greece: CALB-1e-07 (1 ind.), CALA-5e-07 (1 ind.) [coll. 19.9.2007]; CALA-15c-08 (1 ind.), CALA-20b-08 (1 ind.), CALB-20b-08 (1 ind.), CALA-20c-08 (1 ind.) [coll. 17.6.2008]. Elounda, Crete, Greece: CELA15b-07 (1 ind.), CELA-15e-07 (3 ind.), CELB-20a-07 (2 ind.) [coll. 26.9.2007]; 
CELA-10a-07 (1 ind.), CELA-10d-07 (1 ind.) [coll. 27.9.2007]; CELA-5c-07 (1 ind.), CELB-5d-07 (1 ind.) [coll. 29.9.2007]; CELA-5b-08 (1 ind.), CELA-5d-08 (2 ind.), CELB-15d-08 (3 ind.) [coll. 12.6.2008].

Type locality. Red Sea.

Distribution. Red Sea. Mediterranean Sea: WB, CB, AD, AS. New record for the Greek coast.

Habitat. Shallow subtidal depths, on biogenic calcareous substrates, among photophilic algae and Posidonia oceanica rhizomes.

Remarks. The species is regarded by many authors (e.g. Augener 1913, Fauvel 1919, Licher 2000) as a synonym of Syllis variegata Grube, 1860. Recent works (e.g. San Martín 2003, Çinar 2005) however, regard the two species as distinct, which is also supported by molecular analyses (Aguado et al. 2007).

\section{Syllis cruzi Núńez and San Martín, 1991}

http://species-id.net/wiki/Syllis_cruzi

Syllis cruzi Núñez and San Martín, 1991: 238, figs 2a-j; Çinar and Ergen 2003: 780, fig. 2.

Typosyllis cruzi: Licher 2000: 169, fig. 75.

Material examined. Alykes, Crete, Greece: CALB-20d-08 (1 ind.) [coll. 17.6.2008]. Elounda, Crete, Greece: CELB-10a-08 (1 ind.) [coll. 11.6.2008].

Type locality. Canary Islands (Atlantic Ocean).

Distribution. North-east Atlantic (Canary Islands), Mediterranean Sea: WB, CB, AD, AS, LB. New record for the Aegean Sea.

Habitat. Until $115 \mathrm{~m}$ depth, on coralligenous substrates, among photophilic algae, endobiont of sponges.

\section{Syllis gerundensis (Alós and Campoy, 1981)}

http://species-id.net/wiki/Syllis_gerundensis

Typosyllis gerundensis Alós and Campoy, 1981: 21, figs 1-3; Campoy 1982: 446, figs 55-56; Licher 2000: 171, fig. 77.

Syllis gerundensis: Çinar and Ergen 2003: 783; San Martín 2003: 419, figs 230231.

Material examined. Alykes, Crete, Greece: CALA-20b-08 (1 ind.), CALB-20b-08 (1 ind.) [coll. 17.6.2008]; CALA-5d-08 (2 ind.) [[coll. 18.6.2008]. Elounda, Crete, Greece: CELB-1e-07 (1 ind.) [coll. 29.9.2007]; CELB-1d-08 (1 ind.), CELA-5d-08 (3 ind.) [coll. 12.6.2008].

Type locality. Columbretes Islands, Spain (western Mediterranean Sea). 
Distribution. Mediterranean Sea: WB, CB, AD, AS, LB. New record for the Aegean Sea.

Habitat. Shallow subtidal depths, on calcareous grounds, sandy bottoms, among Posidonia oceanica rhizomes and photophilic algae, endobiont of sponges.

Syllis jorgei San Martín and López, 2000

http://species-id.net/wiki/Syllis_jorgei

Syllis jorgei San Martín and López, 2000: 430, figs 4-6; San Martín 2003: 382, figs 208-210; Çinar and Ergen 2003: 785.

Typosyllis lutea: Campoy 1982: 428.

Syllis lutea: San Martín 1984b: 370, figs 94-95; Arvanitidis 1994: 101 (Non Syllis lutea Hartmann-Schröder, 1960).

Material examined. Haifa Bay, Israel: ALA-IL-7 (3 ind.) [coll. 11.10.2009]. Alykes, Crete, Greece: CALA-20c-07 (1 ind.) [coll. 18.9.2007], CALB-1a-08 (1 ind.) [coll. 18.6.2008]. Elounda, Crete, Greece: CELB-1a-08 (1 ind.), CELA-1c-08 (1 ind.), CELB-5d-08 (1 ind.) [coll. 12.6.2008].

Type locality. Columbretes Islands, Spain (western Mediterranean Sea).

Distribution. East Atlantic (Canary Islands), Mediterranean Sea: WB, CB, AD, AS, LB. New record for the Israeli coast.

Habitat. Until $145 \mathrm{~m}$ depth (Çinar and Ergen 2003), on biogenic calcareous structures, among Posidonia oceanica rhizomes and photophilic algae.

\section{Syllis pulvinata (Langerhans, 1881)}

http://species-id.net/wiki/Syllis_pulvinata

Typosyllis pulvinata Langerhans, 1881: 97, 104; Licher 2000: 158, fig. 70.

Syllis pulvinata: Çinar and Ergen 2003: 787; San Martín 2003: 372, figs 202-204.

Syllis (Typosyllis) truncata mediterranea Ben-Eliahu, 1977a: 10, fig. 2.

Syllis mediterranea: San Martín, 1984b; 209, fig. 8.

Material examined. Elounda, Crete, Greece: CELA-1b-08 (1 ind.), CELA-5c-08 (2 ind.), CELB-15d-08 (1 ind.) [coll. 12.6.2008].

Type locality. Canary Islands (Atlantic Ocean).

Distribution. North-east Atlantic (Cantabrian Sea to Canary Islands), Red Sea, Mediterranean: WB, CB, AD, AS, LB. New record for the Aegean Sea.

Habitat. Shallow subtidal depths, on calcareous substrates (vermetid reefs), among photophilic algae, endobiont of sponges. 


\section{Syllis tyrrhena (Licher and Kuper, 1998)}

http://species-id.net/wiki/Syllis_tyrrhena

Typosyllis tyrrhena Licher and Kuper, 1998: 228, figs 1-4; Licher 2000: 140, figs 2, 14-16, 62-63; Kuper 2001: 58, figs 1a-b, 20-24. Amaral et al. 2005: 162, figs a-f on same page.

Syllis tyrrhena: San Martín 2003: 379, fig. 207.

Material examined. Elounda, Crete, Greece: CELB-10b-08 (1 ind.) [coll. 11.6.2008]. Type locality. Island of Elba, Italy (western Mediterranean Sea).

Distribution. Brazil (Amaral et al. 2005), Mediterranean Sea: WB, AS. New record for the eastern Mediterranean Sea.

Habitat. Until $13 \mathrm{~m}$ depth, in sandy substrates of mixed grain sizes (Licher and Kuper 1998), on rocks among algae (this study).

\section{Syllis westheidei San Martín, 1984}

http://species-id.net/wiki/Syllis_westheidei

Syllis westheidei San Martín, 1984b: 403, figs 108-109; 2003: 436, figs 240-241; Çinar 1999: 310, fig. 4.141.

Typosyllis westheidei: Licher 2000: 111, fig. 51; Böggemann and Westheide 2004: 418. Typosyllis variegata: Westheide 1974a: 51, figs 21-22. (Non Syllis variegata Grube, 1860).

Material examined. Alykes, Crete, Greece: CALB-15d-08 (1 ind.) [coll. 17.6.2008]. Type locality. Balearic Islands (western Mediterranean Sea).

Distribution. Pacific Ocean (Galápagos Islands), Red Sea, Mediterranean: WB, $\mathrm{CB}, \mathrm{AD}, \mathrm{AS}$. New record for the Greek coast.

Habitat. Shallow subtidal depths, on hard substrates, among photophilic algae, in Posidonia oceanica rhizomes and vermetid reefs.

\section{Genus Trypanosyllis Claparède 1864}

Type species. Syllis zebra Grube, 1860

\section{Trypanosyllis coeliaca Claparède, 1868}

http://species-id.net/wiki/Trypanosyllis_coeliaca

Trypanosyllis coeliaca Claparède 1868: 513, pl. 13, fig. 3; Fauvel 1923: 270, figs 101fh; Cognetti 1957: 27, fig. 5a; 1961: 296, Hartmann-Schröder 1979: 78; Perkins 1981: 1155, figs 33-34; Campoy 1982: 354; Uebelacker 1984: 93, fig. 88; San 
Martín 1984b: 274, fig. 63; 2003: 308, figs 169-170; Arvanitidis 1994: 109; Çinar 1999: 316, fig. 4.144; Çinar and Ergen 2003: 789.

Pseudosyllis brevipennis Grube, 1863: 44, pl. 4, fig. 5.

Material examined. Haifa Bay, Israel, eastern Mediterranean Sea, Station ALA-IL-7 (1 ind.) [coll. 11.10.2009]. Alykes, Crete, Greece: CALA-10b-08 (1 ind.), CALB-10c-08 ( 1 ind.) [coll. 17.6.2008]; CALA-5a-08 (1 ind.), CALB-1d-08 (2 ind.), CALB-5a-08 (1 ind.) [coll. 18.6.2008]. Elounda, Crete, Greece: CELA-15b-07 (1 ind.), CELA15c-07 (1 ind.) [coll. 26.9.2007]; CELB-5c-07 (1 ind.), CELA-10a-07 (1 ind.), CELB-10c-07 (1 ind.) [coll. 27.9.2007]; CELB-1a-07 (2 ind.), CELB-1e-07 (1 ind.) [coll. 29.9.2007]; CELB-10b-08 (1 ind.), CELA-15a-08 (1 ind.) [coll. 17.6.2008]; CELB-1b-08 (1 ind.), CELA-5b-08 (1 ind.), CELA-5c-08 (1 ind.), CELB-5c-08 (1 ind.), CELA-5d-08 (2 ind.) [coll. 18.6.2008].

Type locality. Gulf of Naples (western Mediterranean Sea).

Distribution. Circumtropical. Mediterranean Sea: WB, CB, AD, AS, LB. New record for the Israeli coast.

Habitat. From infralitoral depths to $760 \mathrm{~m}$, on hard substrates, among algae, corals, hydrozoans, sponges and Posidonia oceanica rhizomes, in vermetid reefs, in coarse sand.

Remarks. Specimens from Greece have a faint or no visible trepan. Individuals without trepan but otherwise identical to T. coeliaca have in the past been identified as Pseudosyllis brevipennis Grube, 1863, but according to San Martín (2003) the absence of the trepan can be attributed to a number of reasons, including loss, and $P$. brevipennis is regarded as a synonym of T. coeliaca.

\section{Discussion}

The present study yielded a number of species reported for the first time in the respective areas, and a high number of the new additions belong to the subfamily Exogoninae (Fig. 3). This could be explained by the fact that the small-sized individuals of this subfamily might have been overlooked in earlier works on the syllid fauna of the area which report only very few or no Exogoninae species at all (e.g. Fauvel 1957, Tebble 1959, Bellan 1964, Ergen 1976). The Exogoninae genus Prosphaerosyllis, which has recently been raised from subgeneric to generic level by San Martín (2005), has a difficult and confused taxonomy and several species have recently been described or transferred to the genus (Olivier et al. 2011, Çinar et al. 2011). Currently, 31 species of the genus are considered valid (including an unnamed one, see San Martín 2003), of which 11 have so far been reported to occur in the Mediterranean Sea (Table 3). However, several of the reported species in the area do in fact belong to other species, the identity of which can only be determined through thorough examination of the material in question. The presence of the Red Sea species P. brevicirra (Hartmann-Schröder, 1960) in the Mediterranean belongs to these doubtful records. Records of Sphaerosyllis brevicirra from the western Mediterranean 
Table 3. Reported distribution records of Prosphaerosyllis species in the Mediterranean. $\dagger=$ doubtful record, identity unknown. $\ddagger=$ doubtful record, probably Prosphaerosyllis sp. [unnamed, San Martín 2003], $\S=$ doubtful record, probably $P$. marmarae. References: $1=$ this study, $2=$ San Martín 1984, $3=$ San Martín 2003, 4= Gambi et al. 1995, 5= Alós 1989, 6= Somaschini et al. 1994, 7= Lanera et al. 1990, 8= Zenetos et al. 1997, 9= Simboura 1996, 10= Çinar 1999, 11= San Martín et al. 1982, 12= Çinar and Ergen 2002, 13= Çinar et al. 2003, 14= Somaschini and San Martín 1994, 15= Çinar et al. 2011, 16= Katzmann, 1983, 17= Ben-Eliahu 1977a. Literature-based works (e.g. Musco and Giangrande 2005, Simboura and Nicolaidou 2001) are not included to avoid repetition of records.

\begin{tabular}{|c|c|c|c|c|c|c|}
\hline Species & Type locality & WB & AD & CB & AS & LB \\
\hline P. adelae San Martín, 1984 & $\begin{array}{l}\text { Balearic Islands, Spain, west } \\
\text { Mediterranean }\end{array}$ & 2,3 & & & & 1 \\
\hline $\begin{array}{l}\text { P. brandhorsti (Hartmann- } \\
\text { Schröder, 1965) }\end{array}$ & $\begin{array}{l}\text { Isla Mocha, Chile, Pacific } \\
\text { Ocean }\end{array}$ & $4^{\dagger}$ & & & & \\
\hline $\begin{array}{l}\text { P. brevicirra (Hartmann- } \\
\text { Schröder, 1960) }\end{array}$ & Ghardaqa, Egypt, Red Sea & $\begin{array}{l}4^{\ddagger}, 5^{\ddagger}, \\
6^{\ddagger}, 7^{\ddagger}\end{array}$ & & $8^{\ddagger}$ & $9^{\ddagger}, 10^{\ddagger}$ & \\
\hline $\begin{array}{l}\text { P. campoyi (San Martín et al., } \\
\text { 1982) }\end{array}$ & $\begin{array}{l}\text { Andalusia, Spain, western } \\
\text { Mediterranean }\end{array}$ & 3,11 & & & 1,12 & 13 \\
\hline $\begin{array}{l}\text { P. chauseyensis Olivier et al., } \\
2011\end{array}$ & $\begin{array}{l}\text { Normandy, France, north-east } \\
\text { Atlantic }\end{array}$ & & & & & 1 \\
\hline $\begin{array}{l}\text { P. giandoi (Somaschini and San } \\
\text { Martín, 1994) }\end{array}$ & $\begin{array}{l}\text { Tyrrenian Sea, Italy, western } \\
\text { Mediterranean }\end{array}$ & 14 & & & & \\
\hline $\begin{array}{l}\text { P. longipapillata (Hartmann- } \\
\text { Schröder, 1979) }\end{array}$ & Broome, north-west Australia & & & & & 3,1 \\
\hline P. marmarae Çinar et al., 2011 & $\begin{array}{l}\text { Marmara Sea, Turkey, eastern } \\
\text { Mediterranean }\end{array}$ & & & & 15 & 1 \\
\hline $\begin{array}{l}\text { Prosphaerosyllis sp. [unnamed, } \\
\text { San Martín 2003] }\end{array}$ & $\begin{array}{l}\text { Cabo de Creus, Spain, western } \\
\text { Mediterranean }\end{array}$ & 3 & & & & \\
\hline P. tetralix (Eliason, 1920) & Öresund, Sweden & 3 & 16 & 8 & & $17^{\S}$ \\
\hline $\begin{array}{l}\text { P. xarifae (Hartmann-Schröder, } \\
\text { 1960) }\end{array}$ & Sarso, Egypt, Red Sea & 3 & & & 12 & \\
\hline
\end{tabular}

Sea by Alós (1989) and from the Aegean Sea (Simboura 1996, Çinar 1999) belong to an undescribed Prosphaerosyllis species (San Martín 2003). These differ from P. brevicirra by the absence of dorsal cirri on chaetiger 2 (reported as present in Alós' (1989) description but in fact absent (San Martín 2003)), the absence of the conspicious papilla on the dorsal cirrus and by thicker aciculae. Hartmann-Schröder (1960) does not mention the papilla on the dorsal cirrus in her description of the species (only visible in the illustrations, but confirmed through examination of type material); instead she focuses on the reduced length of the dorsal cirri as a character to distinguish the species from its congeners. This fact might have lead to confusion of $P$. brevicirra with other species possessing short dorsal cirri. Two other reports of the species from adjacent areas (Red Sea, Atlantic) likewise do probably not belong to P. brevicirra: Ben-Eliahu's (1977a) redescription of the species based on material from the Gulf of Elat (Red Sea) differs in several aspects from Hartmann-Schröder's (1960) descrip- 
tion and from the type material. In particular, Ben-Eliahu does not mention or illustrate the papilla on the dorsal cirrus, her specimens have four eyes and one anterior pair of eyespots (eyespots, a character considered as invariable within species (Riser 1991), are absent in P. brevicirra) and the proventriculum occupies 4 chaetigers ( 3 in P. brevicirra). According to the description and illustrations, the species might in fact belong to P. marmarae (see remarks for this species above). The record of Sphaerosyllis brevicirra from the Spanish Atlantic coast (Parapar et al. 1994), though described as similar to Alós' (1989) specimens, differs in fact from these by the presence of dorsal cirri on chaetiger 2 and much longer dorsal cirri. It also differs from $P$. brevicirra in having falcigers with serrated blades in anterior chaetigers, no papilla on the dorsal cirrus and much longer dorsal cirri anteriorly $(140 \mu \mathrm{m}$ vs ca. $20 \mu \mathrm{m}$ in P. brevicirra $)$. The species P. brandhorsti (Hartmann-Schröder, 1965) has been recorded in Italy by Gambi et al. (1995). However, the only other records of the species apart from its type locality (Isla Mocha, Chile) are from the northern Pacific (Banse 1972) and belong possibly to possibly $P$. ranunculus (Kudenov and Harris, 1995). The presence of $P$. brandhorsti in the Mediterranean Sea has thus to be considered as doubtful. An identification key to the currently valid Mediterranean species of Prosphaerosyllis can be found below.

\section{Key to the Mediterranean species of Prosphaerosyllis}

1 Dorsal cirri on chaetiger 2 present 2

- $\quad$ Dorsal cirri on chaetiger 2 absent... Prosphaerosyllis sp. [San Martín 2003]

2 Dorsal cirri and antennae with conspicuous papilla P. chauseyensis

Dorsal cirri and antennae without conspicuous papilla ................................ 3

Papillae on dorsum arranged in regular longitudinal rows . .4 Papillae on dorsum arranged irregularly.... .5 Pharynx through 4-5 chaetigers, pharyngeal tooth on midline of pharynx ... P. longipapillata

Pharynx through 3 chaetigers, pharyngeal tooth in anterior third of pharynx..... Dorsal cirri with bulbous cirrophore and rounded or elongated cirrostyle...7 7 Prostomium retracted under posterior chaetigers. Antennae and dorsal cirri distally truncated. Aciculae subdistally with a crown of spines P. adelae Prostomium not retracted under posterior chaetigers. Antennae and dorsal cirri distally rounded. Aciculae with subdistal swelling P. giandoi Palps densely papillated. Dorsal papillation inconspicious. P. marmarae Palps with few or no papillae. Dorsum with distinct papillation .8 Blades of falcigers in midbody with strong serration P. campoyi Blades of falcigers finely serrated P. xarifae 


\section{Acknowledgements}

The authors acknowledge assistance from the following colleagues: Dr Melih Ertan Çinar (Ege University, Turkey), Dr Angelika Brandt (Zoological Museum, University of Hamburg, Germany) and Dr Tarik Meziane (Muséum National d'Histoire Naturelle) for loans of comparative material of Sphaerosyllis longipapillata, Prosphaerosyllis marmarae, Sphaerosyllis brevicirra, Prosphaerosyllis chauseyensis and Prosphaerosyllis laubieri, Dr Guillermo San Martín (Universidad Autónoma de Madrid) for the provision of some of the literature resources and Dr Lyubomir Penev (Pensoft Publishers, Bulgaria) for support with the publication of data as a Darwin Core Archive. Financial support was provided by ViBRANT (FP7, EU, Contract no. RI-261532). This study forms part of the core biodiversity project of the Institute of Marine Biology and Genetics (HCMR).

\section{References}

Abd-Elnaby FA (2009) New Records of Polychaetes from the South Part of Suez Canal, Egypt. World Journal of Fish and Marine Sciences 1: 7-19. http://idosi.org/wjfms/ wjfms 1\%281\%2909/2.pdf

Abd-Elnaby FA, San Martín G (2010) Eusyllinae, Anoplosyllinae, and Exogoninae (Polychaeta: Syllidae) for the Mediterranean Coasts of Egypt, Together the Description of One New Species. Life Science Journal 7: 132-139. http://www.sciencepub.net/life/life0704/20_40 43life0704_132_139.pdf

Abd-Elnaby FA, San Martín G (2011) Syllinae (Syllidae: polychaeta) from the Mediterranean coast of Egypt with the description of two new species. Mediterranean Marine Science 12: 43-52. http://www.medit-mar-sc.net/files/201101/12-104153421_MMS_v12n1_ABD_ ELNABY.pdf

Aguado MT, Bleidorn C (2010) Conflicting signal within a single gene confounds syllid phylogeny (Syllidae, Annelida). Molecular Phylogenetics and Evolution 55: 1128-1138. doi: 10.1016/j.ympev.2010.01.012

Aguado MT, Nygren A, Siddall ME (2007) Phylogeny of Syllidae (Polychaeta) based on combined molecular analysis of nuclear and mitochondrial genes. Cladistics 23: 552-564. doi: 10.1111/1096-0031.2007.00163.x

Aguado MT, San Martín G (2007) Syllidae (Polychaeta) from Lebanon with two new reports for the Mediterranean Sea. Cahiers de Biologie Marine 48: 207-224. http://www.sb-roscoff.fr $/ \mathrm{cbm} / \mathrm{cbm}$.htm?execution=e1s2\&_eventId=viewarticledetails\&articleId=2476

Aguado MT, San Martín G (2009) Phylogeny of Syllidae (Polychaeta) based on morphological data. Zoologica Scripta 38: 379-402. doi: 10.1111/j.1463-6409.2008.00380.x

Aguado MT, San Martín G, ten Hove HA (2008) Syllidae (Annelida: Polychaeta) from Indonesia collected by the Siboga (1899-1900) and Snellius II (1984) expeditions. Zootaxa 1673: $1-48$. 
Alós C (1989) Adiciones a la fauna de anelidos poliquetos de la peninsula iberica: familia Syllidae. Cahiers de Biologie Marine 30: 329-338.

Alós C, Campoy A (1981) Typosyllis gerundensis n. sp.: nuevo Syllidae (Annelida, Polychaeta) del Mediterraneo. Publicaciones del Departamento de Zoologia, Universidad de Barcelona, Facultad de Biologia 7: 21-27.

Amaral ACZ, Rizzo AE, Arruda EP (2005) Manual de Identificação dos Invertebrados Marinhos da Região Sudeste-Sul do Brasil, volume 1. São Paolo: Edusp, 288 pp. http://books.google. de/books?id=SUOjxxkSIysC

Antoniadou C, Nicolaidou A, Chintiroglou C (2004) Polychaetes associated with the sciaphilic alga community in the northern Aegean Sea: spatial and temporal variability. Helgoland Marine Research 58: 168-182. doi: 10.1007/s10152-004-0182-6

Arvanitidis C (1994) Systematic and bionomic study of the macrobenthic polychaetes (Annelida, Polychaeta) of the North Aegean. PhD Thesis, Thessaloniki, Greece: Aristotle University of Thessaloniki. http://phdtheses.ekt.gr/eadd/handle/10442/5045

Arvanitidis C (2000) Polychaete fauna of the Aegean Sea: inventory and new information. Bulletin of Marine Science 66: 73-96. http://www.ingentaconnect.com/content/umrsmas/ bullmar/2000/00000066/00000001/art00010

Arvanitidis C, Bellan G, Drakopoulos P, Valavanis VD, Dounas C, Koukouras A, Eleftheriou A (2002) Seascape biodiversity patterns along the Mediterranean and the Black Sea: lessons from the biogeography of benthic polychaetes. Marine Ecology Progress Series 244: 139-152. doi: 10.3354/meps244139

Augener H (1913) Polychaeta I. Errantia. Die Fauna Südwest-Australiens. Ergebnisse der Hamburger südwest-australischen Forschungsreise 1905 4: 65-304. http://www.biodiversitylibrary.org/item/31515

Augener H (1918) Polychaeta. Beiträge zur Kenntnis der Meeresfauna Westafrikas 2: 67-625. http://www.biodiversitylibrary.org/ia/beitrgezurkenn02mich

Banse K (1971) A new species, and additions to the descriptions of six other species of Syllides Örsted (Syllidae: Polychaeta). Journal of the Fisheries Research Board of Canada 28: 14691481. doi: 10.1139/f71-226

Banse K (1972) On some species of Phyllodocidae, Syllidae, Nephtyidae, Goniadidae, Apistobranchidae, and Spionidae (Polychaeta) from the Northeast Pacific Ocean. Pacific Science 26: 191-222. http://hdl.handle.net/10125/400

Bellan G (1964) Campagne de la Calypso: Méditerranée Nord-Orientale. 6. Annélides Polychètes. Annales de l'Institut océanographique 41: 271-288.

Ben-Eliahu MN (1976a) Errant polychaete cryptofauna (excluding Syllidae and Nereidae) from rims of similar intertidal vermetid reefs on the Mediterranean coast of Israel and in the Gulf of Elat. Israel Journal of Zoology 25: 156-177.

Ben-Eliahu MN (1976b) Polychaete cryptofauna from rims of similar intertidal vermetid reefs on the Mediterranean coast of Israel and in the Gulf of Elat: Sedentaria. Israel Journal of Zoology 25: 121-156.

Ben-Eliahu MN (1977a) Polychaete cryptofauna from rims of similar intertidal vermetid reefs on the Mediterranean coast of Israel and in the Gulf of Elat: Exogoninae and Autolytinae (Polychaeta Errantia: Syllidae). Israel Journal of Zoology 26: 59-99. 
Ben-Eliahu MN (1977b) Polychaete cryptofauna from rims of similar intertidal vermetid reefs on the Mediterranean coast of Israel and in the Gulf of Elat: Syllinae and Eusyllinae (Polychaeta Errantia: Syllidae). Israel Journal of Zoology 26: 1-58.

Ben-Eliahu MN, Fiege D (1995) Polychaeta from the continental shelf and slope of Israel collected by "Meteor" 5 Expedition (1987). Senckenbergiana maritima 25: 85-105.

Ben-Eliahu MN, Golani D (1990) Polychaetes (Annelida) in the gut contents of goatfishes (Mullidae), with new polychaete records for the Mediterranean coast of Israel and the Gulf of Elat (Red Sea). Marine Ecology 11: 193-205. doi: 10.1111/j.1439-0485.1990. tb00239.x

Blagoderov V, Brake I, Georgiev T, Penev L, Roberts D, Ryrcroft S, Scott B, Agosti D, Catapano T, Smith VS (2010) Streamlining taxonomic publication: a working example with Scratchpads and ZooKeys. ZooKeys 50: 17-28. doi: 10.3897/zookeys.50.539

Böggemann M, Westheide W (2004) Interstitial Syllidae (Annelida: Polychaeta) from Mahe (Seychelles). Journal of Natural History 38: 403-446.

Campoy A (1982) Fauna de Espana de Anelidos Poliquetos de la Peninsula Iberica. Publicaciones de Biologia de la Universidad de Navarra 7: 1-780. http://hdl.handle.net/10171/11773, http://hdl.handle.net/10171/11777

Capa M, San Martín G, López E (2001) Syllinae (Syllidae, Polychaeta) del Parque Nacional de Coiba, Panamá. Revista de Biología Tropical 49: 1-18. http://www.ots.ac.cr/tropiweb/ attachments/volumes/vol49-1/15-Capa-Syllinae-101-114.pdf

Çinar ME (1999) Türkiye’nin Ege Denizi sahillerinde dağılım gösteren syllidae (PolychaetaAnnelida) türlerinin taksonomisi ve ekolojisi. Phd Thesis, Izmir, Turkey: Ege University. http://www.belgeler.com/blg/26fl/turkiye-nin-ege-denizi-sahillerinde-dagilim-gosterensyllidae-polychaeta-annelido-turlerinin-taksonomisi-ve-ekolojisi-taxonomy-and-ecologyof-syllidae-polychaeta-annelido-distrubuted-along-the-turkish-aegeon-coast

Çinar ME (2005) Syllis ergeni: a new species of Syllidae (Annelida: Polychaeta) from Izmir Bay (Aegean Sea, eastern Mediterranean Sea). Zootaxa 1036: 43-53.

Çinar ME, Dagli E, Açik S (2011) Annelids (Polychaeta and Oligochaeta) from the Sea of Marmara, with descriptions of five new species. Journal of Natural History 45: 2105-2143. doi: 10.1080/00222933.2011.582966

Çinar ME, Ergen Z (2002) Faunistic analysis of Syllidae (Annelida: Polychaeta) from the Aegean Sea. Cahiers de Biologie Marine 43: 171-178. http://www.sb-roscoff.fr/cbm/cbm. htm? execution=e1s5\&_eventId=viewarticledetails\&articleId $=3650$

Çinar ME, Ergen Z (2003) Eusyllinae and Syllinae (Annelida: Polychaeta) from northern Cyprus (Eastern Mediterranean Sea) with a checklist of species reported from the Levant Sea. Bulletin of Marine Science 72: 769-793. http://www.ingentaconnect.com/content/ umrsmas/bullmar/2003/00000072/00000003/art00010

Çinar ME, Ergen Z, Benli HA (2003) Autolytinae and Exogoninae (Polychaeta: Syllidae) from northern Cyprus (Eastern Mediterranean sea) with a checklist of species reported from the Levant sea. Bulletin of Marine Science 72: 741-767. http://www.ingentaconnect.com/ content/umrsmas/bullmar/2003/00000072/00000003/art00009

Çinar ME, Gambi MC (2005) Cognetti’s syllid collection (Polychaeta: Syllidae) deposited at the Museum of the Stazione Zoologica "Anton Dohrn" (Naples, Italy), with descrip- 
tions of two new species of Autolytus. Journal of Natural History 39: 725-762. doi: $10.1080 / 00222930400001327$

Çinar ME, Katağan T, Koçak F, Öztürk B, Ergen Z, Kocatas A, Önen M, Kirkim F, Bakir K, Kurt Şahin G, Dağli E, Açik S, Doğan A, Özcan T (2008) Faunal assemblages of the mussel Mytilus galloprovincialis in and around Alsancak Harbour (Izmir Bay, eastern Mediterranean) with special emphasis on alien species. Journal of Marine Systems 71: 1-17. doi: 10.1016/j.jmarsys.2007.05.004

Claparède É (1863) Beobachtungen über Anatomie und Entwicklungsgeschichte wirbelloser Thiere an der Küste von Normandie angestellt. Wilhelm Engelmann Verlag, Leipzig, 172 pp. http://biodiversitylibrary.org/item/40310.

Claparède É (1864) Glanures Zootomiques parmi les Annélides de Port-Vendres (Pyrenées Orientales). Mémoires de la Société de physique et d'histoire naturelle de Genève 17: 463600. http://www.biodiversitylibrary.org/ia/glanureszootomiq00clapare

Claparède É (1868) Les annélides chétopodes du Golfe de Naples. Mémoires de la Société de physique et d'histoire naturelle de Genève 20: 313-584. http://biodiversitylibrary.org/item/18576

Cognetti G (1957) I Sillidi del Golfo di Napoli. Pubblicazioni della Stazione Zoologica di Napoli 30: 1-100.

Cognetti G (1961) Les Syllidiens des côtes de Bretagne. Cahiers de Biologie Marine 2: 291-312. De Matos Nogueira JM, Amaral ACZ, San Martín G (2001) Description of five new species of Exogoninae Rioja, 1925 (Polychaeta: Syllidae) associated with the stony coral Mussismilia hispida (Verrill, 1868) in Sao Paulo State, Brazil. Journal of Natural History 35: 1773-1794. doi: 10.1080/00222930152667096

Day JH (1967) A monograph on the Polychaeta of Southern Africa. Part 1. Errantia. Trustees of the British Museum (Natural History), London, 498 pp. http://www.biodiversitylibrary. org/ia/monographonpolyc01dayj

Ding Z-hu, Westheide W (2008) Interstitial Exogoninae from the Chinese coast (Polychaeta, Syllidae). Senckenbergiana Biologica 88: 125-159.

Eliason A (1920) Polychaeta. Biologisch-faunistische Untersuchungen aus dem Öresund. Acta Universitatis Lundensis 16: 1-103. http://www.biodiversitylibrary.org/ia/biologischfaunis00elia

Ergen Z (1976) Investigations on the taxonomy and ecology of Polychaeta from Izmir Bay and its adjacent area. Scientific Reports of the Faculty of Science Ege University 209: 1-66.

Fassari G (1982) Anellidi Policheti del mar Egeo. Animalia 9: 109-121.

Fauchald K (1977) Polychaetes from intertidal areas in Panama, with a review of previous shallow-water records. Smithsonian Contributions to Zoology 221: 1-81. http://hdl.handle.net/10088/5511 doi: 10.5479/si.00810282.221

Faulwetter S, Chatzigeorgiou G, Galil BS, Nicolaidou A, Arvanitidis C (2011) Sphaerosyllis levantina sp. n. (Annelida) from the eastern Mediterranean, with notes on character variation in Sphaerosyllis hystrix Claparède, 1863. In: Smith V, Penev L (Eds) e-Infrastructures for data publishing in biodiversity science. ZooKeys 150: 327-345. doi: 10.3897/zookeys. 150.1877

Fauvel P (1919) Annélides Polychètes de Madagascar, de Djibouti, et du Golfe Persique. Archives de zoologie expérimentale et générale 58: 315-473. http://www.biodiversitylibrary. org/page/6316667 
Fauvel P (1923) Polychètes errantes. Faune de France, Paris, 488 pp. http://www.faunedefrance. org/bibliotheque/docs/P.FAUVEL(FdeFr05)Polychetes-errantes.pdf

Fauvel P (1955) Contribution a la faune des Annelides Polychetes des côtes d'Israel. I. Bulletin of the Sea Fisheries Research Station, Haifa 10: 3-12.

Fauvel P (1957) Contribution a la faune des Annelides Polychetes des côtes d'Israel. II. Bulletin of the Research Council of Israel 6B: 213-219.

Fukuda MV, Yunda-Guarin G, Nogueira JMM (2009) The genus Prosphaerosyllis (Polychaeta: Syllidae: Exogoninae) in Brazil, with description of a new species. Journal of the Marine Biological Association of the United Kingdom 89: 1443-1454. doi: 10.1017/ S0025315409000095

Gambi MC, Giangrande A, Martinelli M, Chessa LA (1995) Polychaetes of a Posidonia oceanica bed off Sardinia (Italy): Spatio-temporal distribution and feeding guild analysis. Scientia Marina 59: 129-141. http://www.icm.csic.es/scimar/index.php/secId/6/IdArt/2722

Gidholm L (1967) A revision of Autolytinae (Syllidae, Polychaeta) with special reference to Scandinavian species, and with notes on external and internal morphology, reproduction and ecology. Arkiv för Zoologi 19: 157-213.

Goodrich ES (1930) On a new hermaphrodite Syllid. Quarterly Journal of Microscopical Science, London 73: 651-666. http://jcs.biologists.org/content/s2-73/292/651.full.pdf

Gravier CJ (1900) Contribution à l'étude des Annélides Polychètes de la Mer Rouge. Première partie. Nouvelles Archives du Museum d'Histoire Naturelle Paris 2: 137-282.

Grube AE (1850) Die Familien der Anneliden. Archiv für Naturgeschichte 16: 249-364. http://www.biodiversitylibrary.org/ia/diefamilienderan00grub

Grube AE (1860) Beschreibung neuer oder wenig bekannter Anneliden. Beitrag: Zahlreiche Gattungen. Archiv für Naturgeschichte, 26: 71-118. http://www.biodiversitylibrary.org/ page/7153453

Grube AE (1863) Beschreibung neuer oder wenig bekannter Anneliden. Beitrag: Zahlreiche Gattungen. Archiv für Naturgeschichte 29: 37-69. http://www.biodiversitylibrary.org/ page/7071934

Harlock R, Laubier L (1966) Notes on Branchiosyllis uncinigera (Hartmann-Schröder, 1960) new to the Mediterranean. Israel Journal of Zoology 15: 18-25.

Hartmann-Schröder G (1956) Polychaeten-Studien I. Zoologischer Anzeiger 157: 87-91.

Hartmann-Schröder G (1960) Polychaeten aus dem Roten Meer. Kieler Meeresforschungen 16: 69-125.

Hartmann-Schröder G (1962) Zur Kenntnis des Eulitorals der chilenischen Pazifikküste und der argentinischen Küste Südpatagoniens unter besonderer Berücksichtigung der Polychaeten und Ostracoden. Mitteilungen aus dem Hamburgischen zoologischen Museum und Institut 60: 57-270.

Hartmann-Schröder G (1965) Zur Kenntnis des Sublitorals der chilenischen Küste unter besonderer Berücksichtigung der Polychaeten und Ostracoden. Mitteilungen aus dem Hamburgischen zoologischen Museum und Institut 62: 59-305.

Hartmann-Schröder G (1979) Die Polychaeten der tropischen Nordwestküste Australiens (zwischen Derby im Norden und Port Hedland im Süden). Mitteilungen aus dem Hamburgischen zoologischen Museum und Institut 76: 77-218. 
Hartmann-Schröder G (1980a) Die Polychaeten der Amsterdam-Expeditionen nach Westindien. Bijdragen tot de dierkunde 50: 387-401.

Hartmann-Schröder G (1980b) Die Polychaeten der tropischen Nordwestküste Australiens (zwischen Port Samson im Norden und Exmouth im Süden). Mitteilungen aus dem Hamburgischen zoologischen Museum und Institut 77: 41-110.

Hartmann-Schröder G (1981) Die Polychaeten der tropisch-subtropischen Westküste Australiens (zwischen Exmouth im Norden und Cervantes im Süden). Mitteilungen aus dem Hamburgischen zoologischen Museum und Institut 78: 19-96.

Hartmann-Schröder G(1982) Die Polychaeten der subtropisch-antiborealen Westküste Australiens (zwischen Cervantes im Norden und Cape Naturaliste im Süden). Mitteilungen aus dem Hamburgischen zoologischen Museum und Institut 79: 51-118.

Hartmann-Schröder G (1984) Die Polychaeten der antiborealen Südküste Australiens (zwischenAlbany im Westen und Ceduna im Osten). Mitteilungen aus dem Hamburgischen zoologischen Museum und Institut 81: 7-62.

Hartmann-Schröder G (1985) Die Polychaeten der antiborealen Südküste Australiens (zwischen Port Lincoln im Westen und Port Augusta im Osten). Mitteilungen aus dem Hamburgischen zoologischen Museum und Institut 82: 61-99.

Hartmann-Schröder G (1986) Die Polychaeten der antiborealen Südküste Australiens (zwischen Wallaroo im Westen und Port MacDonnell im Osten). Mitteilungen aus dem Hamburgischen zoologischen Museum und Institut 83: 31-70.

Hartmann-Schröder G (1990) Die Polychaeten der subtropisch-tropischen und tropischen Ostküste Australiens zwischen Lake Macquarie (New South Wales) im Süden und Gladstone (Queensland) im Norden. Mitteilungen aus dem Hamburgischen zoologischen $\mathrm{Mu}$ seum und Institut 87: 41-87.

Hartmann-Schröder G (1991) Die Polychaeten der subtropisch-tropischen bis tropischen Ostküste Australiens zwischen Maclean (New South Wales) und Gladstone (Queensland) sowie von Heron Island (Grosses Barrier Riff). Mitteilungen aus dem Hamburgischen zoologischen Museum und Institut 88: 17-71.

Hartmann-Schröder G (1996) Annelida, Borstenwürmer, Polychaeta. 2., neubearbeitete Auflage. Gustav Fischer Verlag, Jena, 648 pp.

Iken K, Konar B (2003) Natural Geography in Nearshore areas (NaGISA): The nearshore component of the Census of Marine Life. Gayana 67: 153-160. doi: 10.4067/S071765382003000200004

Imajima M (1966) The Syllidae (polychaetous annelids) from Japan. III. Eusyllinae. Publications of the Seto Marine Biological Laboratory 14: 85-116.

Imajima M (2003) Polychaetous Annelids from Sagami Bay and Sagami Sea collected by the Emperor Showa of Japan and deposited at the Showa Memorial Institute, National Science Museum, Tokyo (II), Orders included within the Phyllodocida, Amphinomida, Spintherida and Eunicida. National Science Museum Monographs 23: 1-221. http://ci.nii.ac.jp/ naid/110004708004

Jiménez M, San Martín G, López E (1994) Redescription of Pionosyllis neapolitana Goodrich, 1930 and Pionosyllis nutrix Monro, 1936, referred to the genus Grubeosyllis Verrill, 1900 (Polychaeta, Syllidae, Exogoninae). Polychaete Research 16: 52-55. 
Karhan SÜ, Kalkan E, Simboura N, Mutlu E, Bekbölet M (2008) On the occurrence and established populations of the alien polychaete Polydora cornuta Bosc, 1802 (Polychaeta: Spionidae) in the Sea of Marmara and the Bosphorus Strait (Turkey). Mediterranean Marine Science 9: 5-19. http://www.medit-mar-sc.net/files/200812/15-18480210.pdf

Kudenov, JD, Harris LH (1995) Family Syllidae Grube, 1850. In: Blake et al. (Eds) Taxonomic Atlas of the Bentic Fauna of the Santa Maria Basin and Western Santa Barbara Channel. The Annelida Part 2 - Polychaeta: Phyllodocida (Syllidae and Scale-Bearing Families), Amphinomida and Eunicida. Santa Barbara Museum of Natural History, Santa Barbara, 1-97.

Kuper M (2001) Ultrastrukturuntersuchungen der Segmentalorgane, der Spermien und der Brutpflegestrukturen innerhalb der Syllidae (Annelida: Polychaeta). Osnabrück, Germany: Universität Osnabrück. http://d-nb.info/964507099/34

Lamarck J-B de (1818) Histoire naturelle des Animaux sans Vertèbres, préséntant les caractères généraux et particuliers de ces animaux, leur distribution, leurs classes, leurs familles, leurs genres, et la citation des principales espèces qui s'y rapportent; précédée d'une Int. Déterville \& Verdière, Paris, 612 pp. http://www.lamarck.cnrs.fr/ice/ice_book_detail-fr-textlamarck-ouvrages_lamarck-38-1.html

Lanera P, Sordino P, Gambi MC (1990) Annellidi Policheti nuovi o poco conoscuiti per le coste italiane. Oebalia 16: 693-695.

Langerhans P (1879) Die Wurmfauna von Madeira. Zeitschrift für wissenschaftliche Zoologie 32: 513-592.

Langerhans P (1881) Über einige canarische Anneliden. Nova Acta der Kaiserlichen LeopoldCarolin Deutschen Akademie der Naturforscher 42: 95-124.

Langerhans P (1884) Die Wurmfauna von Madeira. IV. Zeitschrift für wissenschaftliche Zoologie 40: 247-285.

Licher F (2000) Revision der Gattung Typosyllis Langerhans, 1879 (Polychaeta: Syllidae). Morphologie, Taxonomie und Phylogenie. Abhandlungen der Senckenbergischen Naturforschenden Gesellschaft 551: 1-336.

Licher F, Kuper M (1998) Typosyllis tyrrhena (Polychaeta, Syllidae, Syllinae), a new species from the island Elba, Tyrrhenian Sea. Italian Journal of Zoology 65: 227-233. doi: $10.1080 / 11250009809386750$

Lińero-Arana I, Díaz Díaz OF (2011) Syllidae (Annelida: Polychaeta) From The Caribbean Coast Of Venezuela. ZooKeys 117: 1-28. doi: 10.3897/zookeys.117.858

López E, San Martín G (1997) Eusyllinae, Exogoninae and Autolytinae (Syllidae, Annelida, Polychaeta) from the Chafarinas Islands (Alboran Sea, W Mediterranean. Miscellània Zoològica 20: 101-111. www.raco.cat/index.php/Mzoologica/article/viewFile/90383/145375

López E, San Martín G, Jiménez M (1996) Syllinae (Syllidae, Annelida, Polychaeta) from Chafarinas Islands (Alborán Sea, W Mediterranean). Miscellània Zoològica 19: 105-118. http://www.raco.cat/index.php/Mzoologica/article/viewFile/90438/145332

Malaquin A (1893) Recherches sur les Syllidiens. Morphologie, anatomie, reproduction, développement. Mémoires de la Société des sciences, de l'agriculture et des arts de Lille 18: 1-477.

McIntosh WC (1885) Report on the Annelida Polychaeta collected by H.M.S. Challenger during the years 1873-1876. Report on the Scientific Results of the Voyage of H.M.S. Chal- 
lenger during the years 1872-76 12: 1-554. http:/www.19thcenturyscience.org/HMSC/ HMSC-Reports/Zool-34/htm/doc.html

Men F, Ding Z-hu, Zhao J, Wu B-L (1993) A preliminary study on small syllides from the Huanghai Sea (Yellow Sea). Journal of Oceanography of Huanghai and Bohai Seas 11: 19-36.

Milne Edwards H (1845) Observations sur le developpement des annelides. Annales des Sciences Naturelles, Paris 3: 145-182. http://biodiversitylibrary.org/page/13408583

Monro CCA (1937) A note on a collection of Polychaeta from the eastern Mediterranean with the description of a new species. Annals and Magazine of Natural History. Series 10 19: 82-86. doi: 10.1080/00222933708655241

Moore JP (1908) Some polychaetous annelids of the northern Pacific coast of North America. Proceedings of the Academy of Natural Sciences of Philadelphia 60: 321-364. http://biodiversitylibrary.org/page/24597271

Moore JP (1909) The polychaetous annelids dredged by the U.S.S. "Albatross" off the coast of southern California in 1904. I. Syllidae, Sphaerodoridae, Hesionidae and Phyllodocidae. Proceedings of the Academy of Natural Sciences of Philadelphia 1909: 321-351. http:// biodiversitylibrary.org/page/26288382

Musco L, Giangrande A (2005) Mediterranean Syllidae (Annelida: Polychaeta) revisited: biogeography, diversity and species fidelity to environmental features. Marine Ecology Progress Series 304: 143-153. doi: 10.3354/meps304143

Nygren A (2004) Revision of Autolytinae (Syllidae: Polychaeta). Zootaxa 680: 1-314.

Núñez J, San Martín G (1991) Two new species of Syllidae (Polychaeta) from Tenerife (Canary Island, Spain). Bulletin of Marine Science 48: 236-241. http://www.ingentaconnect.com/ content/umrsmas/bullmar/1991/00000048/00000002/art00010

Núñez J, San Martín G (1996) Anélidos poliquetos de las Islas Canarias. Familia Syllidae. I. Subfamilias Eusyllinae y Autolytinae. In: Llinás Gonzales et al. (eds) I Congresso de Oceanografía y Recursos Marinos en el Atlántico Centro-Oriental (ICCM). Las Palmas, Gran Canaria (Spain), November 1990. Cabildo Insular de Gran Canaria, Spain, 197-217. http://mdc.ulpgc.es/cdm4/item_viewer.php?CISOROOT=/MDC\&CISOPTR=112681

Núñez J, San Martín G, Carmen Brito M Del (1992) Exogoninae (Polychaeta: Syllidae) from the Canary Islands. Scientia Marina 56: 43-52. http://www.icm.csic.es/scimar/index.php/ secId/6/IdArt/2584/

Olivier F, Grant C, San Martín G, Archambault P, McKindsey CW (2011) Syllidae (Annelida: Polychaeta: Phyllodocida) from the Chausey Archipelago (English Channel, France), with a description of two new species of the Exogoninae Prosphaerosyllis. Marine Biodiversity: Online First. doi: 10.1007/s12526-011-0092-1

Ørsted AS (1845) Fortegnelse over Dyr, samlede i Christianiafjord ved Drobak fra 21-24 Juli, 1844. Naturhistorisk Tidsskrift, Köbenhavn 1: 400-427.

Parapar J, San Martín G, Besteiro C, Urgorri V (1994) Aspectos sistemáticos y ecológicos de las Subfamilias Eusyllinae y Exogoninae (Polychaeta, Syllidae) en la Ría de Ferrol (Galicia, NO España). Boletín de la Real Sociedad Española de Historia Natural 91: 91-101.

Penev L, Agosti D, Georgiev T, Catapano T, Miller J, Blagoderov V, Roberts D, Smith VS, Brake I, Ryrcroft S, Scott B, Johnson NF, Morris RA, Sautter G, Chavan V, Robertson T, 
Remsen D, Stoev P, Parr C, Knapp S, Kress WJ, Thompson FC, Erwin T (2010) Semantic tagging of and semantic enhancements to systematics papers: ZooKeys working examples. ZooKeys 50: 1-16. doi: 10.3897/zookeys.50.538

Penev L, Mietchen D, Chavan VS, Hagedorn G, Remsen DP, Smith VS, Shotton D (2011) Pensoft Data Publishing Policies and Guidelines for Biodiversity Data. Available at: http:// www.pensoft.net/J_FILES/Pensoft_Data_Publishing_Policies_and_Guidelines.pdf

Perkins TH (1981) Syllidae, principally from Florida, with descriptions of a new genus and twenty-one new species. Proceedings of the Biological Society of Washington 93: 10801172. http://biostor.org/reference/73932

Pierantoni U (1903) La gestazione esterna. Contributo alla biologia ed all embriologia dei Sillidi. Archivio Zoologico Italiano 1: 231-252. http://biodiversitylibrary.org/page/5747694 Por FD (1989) The legacy of Tethys: an aquatic biogeography of the Levant. Kluwer Academic Publishers, Dordrecht, 216 pp.

Ramos J, San Martín G, Sikorski A (2010) Syllidae (Polychaeta) from the Arctic and subArctic regions. Journal of the Marine Biological Association of the United Kingdom 90: 1041-1050. doi: 10.1017/S0025315409991469

Riser NW (1991) An evaluation of taxonomic characters in the genus Sphaerosyllis (Polychaeta: Syllidae). Ophelia supplement: 209-218.

Rullier F (1972) Annélides polychètes de Nouvelle-Calédone. Expédition Française sur les Récifs Coralliens de la Nouvelle-Calédonie 6: 1-167.

Russell DE (1991) Exogoninae (Polychaeta: Syllidae) from the Belizean barrier reef with a key to species of Sphaerosyllis. Journal of Natural History 25: 49-74. doi: $10.1080 / 00222939100770061$

Ruíz-Ramírez JD, Salazar-Vallejo SI (2001) Exogoninae (Polychaeta: Syllidae) del Caribe mexicano con una clave para las especies del Gran Caribe. Revista de Biología Tropical 49: 117-140. http://www.scielo.sa.cr/scielo.php?pid=S0034-77442001000100012

Saint-Joseph A de (1887) Les Annelides polychetes des cotes de Dinard, pt. 1. Annales des sciences naturelles 1: 127-270. http://biodiversitylibrary.org/page/33074463

San Martín G (1984a) Descripcion de una nueva especie y revision del genero Sphaerosyllis (Polychaeta: Syllidae). Cahiers de Biologie Marine 25: 375-391.

San Martín G (1984b) Estudio biogeográfico, faunistico y sistemático de los poliquetos de la familia Silidos (Syllidae: Polychaeta) en Baleares. PhD Thesis, Madrid, Spain: Universidad Complutense de Madrid.

San Martín G (1991a) Grubeosyllis and Exogone (Exogoninae, Syllidae, Polychaeta) from Cuba, the Gulf of Mexico, Florida and Puerto Rico, with a revision of Exogone. Bulletin of Marine Science 49: 715-740. http://www.ingentaconnect.com/content/umrsmas/bull$\mathrm{mar} / 1991 / 00000049 / 00000003 / \mathrm{art} 00004$

San Martín G (1991b) Sphaerosyllis and Parapionosyllis (Polychaeta: Syllidae) from Cuba and Florida. Ophelia supplement 5: 231-238.

San Martín G (1994) Autolytinae (Polychaeta, Syllidae) from Cuba and north American Atlantic Ocean. Mémoires du Muséum national d'Histoire Naturelle 162: 269-277.

San Martín G (2003) Annelida, Polychaeta II: Syllidae. In: Ramos MA et al. (Eds) Fauna Ibérica, vol. 21. Museo Nacional de Ciencias Naturales. CSIC, Madrid, 554 pp. 
San Martín G (2005) Exogoninae (Polychaeta: Syllidae) from Australia with the description of a new genus and twenty-two new species. Records of the Australian Museum 57: 39152. http://publications.australianmuseum.net.au/pdf/1438_complete.pdf doi: 10.3853/ j.0067-1975.57.2005.1438

San Martín G (2008) Syllinae (Polychaeta: Syllidae) from Australia. Part 1. Genera Branchiosyllis, Eurysyllis, Karroonsyllis, Parasphaerosyllis, Plakosyllis, Rhopalosyllis, Tetrapalpia n.gen., and Xenosyllis. Records of the Australian Museum 60: 119-160. http://australianmuseum.net. au/Uploads/Journals/18066/1494_complete.pdf doi: 10.3853/j.0067-1975.60.2008.1494

San Martín G, Acero MI, Contonente M, Gomez JJ (1982) Una coleccion de anelidos poliquetos de las costas mediterraneas andaluzas. Actas do II Simposio Iberico de Estudos do Benthos Marinho, Liboa 3: 171-182.

San Martín G, Alvarado R (1981) Nota sobre poliquetos de la isla de Cabrera (Baleares). Boletín de la Real Sociedad Española de Historia Natural 79: 221-234.

San Martín G, Bone D (2001) Syllidae (Polychaeta) de praderas de Thalassia testudinum en el Parque Nacional Morrocoy (Venezuela). Revista de Biología Tropical 49: 609-620. http:// www.scielo.sa.cr/scielo.php?pid=S0034-77442001000200019

San Martín G, Gonzalez G, López E (1985) Aspectos sistematicos y ecologicos sobre algunas especies de Silidos (Polychaeta: Syllidae) de las costas gallegas. Boletin Instituto Espanol de Oceanografia 2: 27-36.

San Martín G, Hutchings PA (2006) Eusyllinae (Polychaeta: Syllidae) from Australia with the description of a new genus and fifteen new species. Records of the Australian Museum 58: 257-370. http://publications.australianmuseum.net.au/pdf/1466_complete.pdf doi: 10.3853/j.0067-1975.58.2006.1466

San Martín G, López E (2000) Three new species of Syllis (Syllidae: Polychaeta) from Iberian coasts. Cahiers de Biologie Marine 41: 425-433. http://www.sb-roscoff.fr/cbm/cbm. htm?execution=e1s2\&_eventId=viewarticledetails\&articleId=2666

San Martín G, López E, Aguado MT (2009) Revision of the genus Pionosyllis (Polychaeta: Syllidae: Eusyllinae), with a cladistic analysis, and the description of five new genera and two new species. Journal of the Marine Biological Association of the United Kingdom 89: 1455 doi: 10.1017/S0025315409003099

Sardá R (1984) La subfamilia Exogoninae (Polychaeta, Syllidae) de Gibraltar, con la descripción de Pseudobrania euritmica n. sp. Publicaciones del Departamento de Zoologia, Universidad de Barcelona, Facultad de Biologia 10: 7-13.

Simboura N (1996) Marine macrobenthic Polychaetes (Annelida, Polychaeta) of Greece: Taxonomy, Ecology, Zoogeography. PhD Thesis, Athens, Greece: University of Athens. http:// phdtheses.ekt.gr/eadd/handle/10442/5952

Simboura N, Nicolaidou A (2001) The Polychaetes (Annelida, Polychaeta) of Greece: Checklist, Distribution and Ecological Characteristics. Monographs on Marine Sciences 4: 1-115.

Somaschini A, Gravina MF, Ardizzone GD (1994) Polychaete Depth Distribution in a Posidonia oceanica Bed (Rhizome and Matte Strata) and Neighbouring Soft and Hard Bottoms. Marine Ecology 15: 133-151. doi: 10.1111/j.1439-0485.1994.tb00049.x

Somaschini A, San Martín G (1994) Description of two new species of Sphaerosyllis (Polychaeta: Syllidae: Exogoninae) and first report of Sphaerosyllis glandulata for the Mediterranean Sea. Cahiers de Biologie Marine 35: 357-367. 
Southern R (1914) Clare Island Survey. Archiannelida and Polychaeta. Proceedings of the Royal Irish Academy 31: 1-160. http://biodiversitylibrary.org/page/34773787

Surugiu V (2005) Inventory of inshore polychaetes from the Romanian coast. Mediterranean Marine Science 6: 51-74. http://www.medit-mar-sc.net/files/200812/15-1718395.pdf

Tebble N (1956) The polychaete fauna of the Gold Coast. Bulletin of the British Museum

(Natural History) Zoology 3: 59-148. http://biodiversitylibrary.org/page/2243542

Tebble N (1959) On a collection of Polychaetes from the Mediterranean coast of Israel. Bulletin of the Research Council of Israel B8: 9-30.

Uebelacker JM (1984) Family Syllidae Grube, 1850. In: Uebelacker JM, Johnson PG (Eds) Taxonomic Guide to the Polychaetes of the Northern Gulf of Mexico, Volume V. Barry A. Vittor \& Associates, Alabama, 30.31-30.151. http://biodiversitylibrary.org/page/3248872

Westheide W (1974a) Interstitielle Fauna von Galapagos. XI. Pisionidae, Hesionidae, Pilargidae, Syllidae (Polychaeta). Mikrofauna des Meeresbodens 44: 1-146.

Westheide W (1974b) Interstitielle Polychaeten aus brasilianischen Sandstränden. Mikrofauna des Meeresbodens 31: 1-16.

Zenetos A, Christianidis S, Pancucci MA, Simboura N, Tziavos C (1997) Oceanologic study of an open coastal area in the Ionian Sea with emphasis on its benthic fauna and some zoogeographical remarks. Oceanologica Acta 20: 437-451.

\section{Supplement information}

The Scratchpads version of this publication is available at: http://polychaetes.marbigen.org/node/1636

Figures are available at: http://polychaetes.marbigen.org/category/image-galleries/eastern-mediterranean-syllidae

Locations and their description are available at: http://polychaetes.marbigen.org/content/darwincorelocation

Tables with species occurences are available at: http://polychaetes.marbigen.org/content/species-occurences-sampling-stations http://polychaetes.marbigen.org/content/reported-distribution-records-prosphaerosyllis-species-mediterranean

The data underpinning the analysis reported in this paper are deposited at:

GBIF (Global Biodiversity Information Facility): http://ipt.pensoft.net/ipt/resource.do?r=easternmedsyllids

Dryad Data Repository: doi: 10.5061/dryad.4b7k408g 